\title{
Multicolor Polarimetry of Selected Be Stars: 1995-98
}

\author{
David McDavid \\ Limber Observatory, Timber Creek Road, P.O. Box 63599, Pipe Creek TX 78063-3599; \\ mcdavid@limber.org
}

\begin{abstract}
A new polarimeter called AnyPol has been used at Limber Observatory for four years to annually monitor the broadband linear polarization of a sample of bright northern Be stars. This is the fourth report on a program started in 1985 at McDonald Observatory and the first one to come entirely from the new installation. Although no variability was detected at the $3 \sigma$ level during the current reporting period, analysis of the full 13-year data set is beginning to reveal hints of long-term variability that may provide clues for understanding the Be phenomenon.
\end{abstract}

Key words: stars: emission-line,Be — stars: mass-loss — stars: evolution instrumentation: polarimeters — techniques: polarimetric

\section{INTRODUCTION}

Be stars are non-supergiant B-type stars whose spectra have, or had at some time, one or more Balmer lines in emission. The mystery of the Be phenomenon is that the emission, which is well understood to originate from a flattened circumstellar envelope or disk, can come and go episodically on time scales of days to decades. This has yet to be explained as a predictable consequence of stellar evolution theory, although many contributing factors have been discussed, including rapid rotation, radiation-driven stellar winds, nonradial pulsation, flarelike magnetic activity, and binary interaction. For the unfamiliar reader, the review of Be stars by Slettebak (1988) will provide an excellent introduction.

Recent optical interferometry combined with spectropolarimetry has directly confirmed that the circumstellar envelopes surrounding Be stars are equatorially flattened (Quirrenbach et al. 1997). Given the small observed polarization of only about 1\%, Monte Carlo computer simulations of polarization by electron scattering of the starlight in the circumstellar envelope (Wood, Bjorkman, \& Bjorkman 1997) constrain it to be an extremely thin disk, with opening angle (half width in latitude) on the order of $3^{\circ}$. This example shows that polarimetry is a very useful observational technique for studying physical properties of the envelopes, with the ultimate purpose of understanding their origin. Toward this end it is of great interest to measure polarization variations and the time scales on which they occur, in order to characterize the physical processes involved. 
This paper is the fourth status report on an ongoing program of annual polarimetric monitoring of a sample of bright northern Be stars begun in 1985 (McDavid 1994 and references therein). The program stars are listed in Table 1, with visual magnitudes taken from Hoffleit and Jaschek (1982) and spectral types and $v \sin i$ values from Slettebak (1982). A broadband filter system (Table 2) was chosen to extend the time base of continuous systematic observations for the study of long-term variability, taking advantage of earlier work which dates back to the 1950s. After 10 years on the $0.9 \mathrm{~m}$ telescope at McDonald Observatory the project was relocated to a new polarimeter on the $0.4 \mathrm{~m}$ telescope at Limber Observatory, where more flexible scheduling allows the study of variability on a greater variety of time scales. Advancements in commercially available instrumentation have made it possible to do so without compromising the quality of the data.

\section{ANYPOL: A GENERIC LINEAR POLARIMETER}

AnyPol got its name from the fact that it is completely generic and incorporates no new principles of design or construction. In most respects, including the color system as specified by prescription, it is a simplified and miniaturized version of the McDonald Observatory polarimeter (Breger 1979): a rapidly rotating Glan-Taylor prism as an analyzer, followed by a Lyot depolarizer, Johnson/Cousins UBVRI glass filters, a Fabry lens, and an uncooled S-20 photomultiplier tube. It is very compact, with stepper motors and belt drives for separate analyzer and filter wheel modules which fit into a single main head unit. A simple postviewer using a right-angle prism at one position of the filter wheel is adequate for finding and centering, and three aperture sizes are available in a slide mechanism with a pair of LEDs for backlighting.

The control system for AnyPol is based on a $66 \mathrm{MHz} 486 \mathrm{PC}$ with plugin multichannel analyzer and stepper motor controller cards, interfaced to the polarimeter head through an electronics chassis unit containing the power supplies and microstepping drivers. A point-and-click display serves to control all selectable functions and parameters and also shows $10 \mathrm{~s}$ updates of the measurement in progress, including a graph of the data buffer. The mathematical details of the data processing were adapted from the control program for the Minipol polarimeter of the University of Arizona (Frecker \& Serkowski 1976).

On the $0.4 \mathrm{~m}$ telescope at Limber Observatory the photon count rates are comparable to those obtained with the $0.9 \mathrm{~m}$ telescope at McDonald Observatory with a neutral density filter of $10 \%$ transmission which was necessary for the bright stars $(V=2-5)$ in the Be star monitoring program. Observational error estimates are derived from the repeatability of multiple independent measurements, and they are in general agreement with checks based on the residuals in the fit to the modulated signal and the uncertainty derived from photon counting statistics. Typical errors are on the order of $0.05 \%$ in the degree of polarization and $2^{\circ}$ in position angle. One of the main

sources of error is a slight variation in the speed of the motor driving the analyzer, which becomes significant at the level of a few hundredths of a percent. All observations are corrected for an 
instrumental polarization on the order of $0.10 \%$, tracked by repeated observations of unpolarized standard stars from the list of Serkowski (1974). The position angle is calibrated by observing polarized standard stars from the list of Hsu \& Breger (1982).

\section{OBSERVATIONS}

The targets for the annual monitoring program were selected to include Be stars with a variety of different characteristics and with the longest possible history of continuous observation. They fall into summer and winter groups, so the basic observing strategy is to make the polarization measurements during about one week in summer and one week in winter. The only interruption in the project has been the loss of 1994 while the new polarimeter was under construction.

One individual measurement consists of 3 cycles through all 5 filters with a 200 s integration time on each filter. If there is a bright Moon, a sky cycle is taken to correct for the background polarization. The result for each single filter is taken to be the mean and standard deviation of the 3 integrations in that filter. (The standard deviation is a more conservative error estimate than the standard deviation of the mean, but it may be more realistic because 3 measurements is a very small sample.) During a typical observing run for this project, about 3 to 5 observations of each program star are collected on different nights during the same week.

\section{ANALYSIS OF VARIABILITY}

The first goal in analyzing the data is to identify clear cases of variable polarization over the time scales covered by this installment of the project. The data are presented in Tables 3-12, which begin with the month and year of the observing run and the number of measurements in each filter. The $q$ and $u$ normalized Stokes parameters, the degree of polarization $p$, and the polarization position angle $\theta$ are all given as the mean and standard deviation for each run. The last column shows the average error in $p$ and $\theta$ for a single measurement. In addition to the program Be stars, two polarized standard stars were also observed as checks on the stability of the system: 2H Cam (HD 21291, HR 1035, $V=4.21$, B9 Ia) in winter and o Sco (HD 147084, HR $6081, V=4.54$, A5 II) in summer.

As in previous papers in this series, summary tables (Tables 13-15) were constructed giving the means and standard deviations of the measurements for each star in each filter over the four annual data sets. The quantities in angled brackets are also four-year averages, and the quantities in rows labeled "GAV" for "grand average" are averages over all five filters.

Since $p$ and $\theta$ carry a statistical bias, $q$ and $u$ are more appropriate quantities for evaluating variability (Clarke \& Stewart 1986). Nevertheless, it may provide more physical insight to study

$p$ and $\theta$ to see if the variability is mainly in polarization degree or position angle. In fact, with the 
limited number of measurements at hand, statistical tests must be applied with caution regardless of which set of parameters is used.

We can apply various simple $3 \sigma$ criteria to conservatively identify variability, as in previous papers in this series. Looking for night-to-night variability, we see that Tables 3-12 show only two cases in which $d q$ or $d u$ is greater than $3 d p i$ : $d u^{B}$ of $\gamma$ Cas in 01/96 and $d u^{B}$ of o And in 06/95. Both cases are negligible, since they occur in only one filter and during only one observing run. For year-to-year variability, Tables $13-15$ show not a single case in which $d q$ or $d u$ is greater than $3<d p i>$. The conclusion is that we can demonstrate no statistically significant variability in the polarization of any of the program stars over the latest 4-year time period.

Since variable polarization was detected in the two previous reports on this project, it seems advisable to search for an explanation by comparing the Limber Observatory system with the system used at McDonald Observatory. For this purpose Table 13 of McDavid (1994) is reproduced here as Table 16 for direct comparison with the present Table 15. These observations of polarized standard stars show very clearly that the two systems match extremely well. The only outstanding difference is the typical precision of a single observation, which is higher in the McDonald system. This is readily understood since the McDonald estimates were based on theoretical photon counting statistics, while the Limber estimates are based on experimental scatter in repeated measurements. With a larger value for the error in a single observation, the Limber system is sometimes a less sensitive detector of variability, but it may also give more realistic results.

Work is underway to make a complete data set available at the Strasbourg astronomical Data Center (CDS), including all of the annual observations from both McDonald Observatory and Limber Observatory published to date in this series of papers. The times will be given in decimal years for better precision than the current specification of month and year.

\section{DISCUSSION}

The $3 \sigma$ criterion used here and in the previous papers of this series is very conservative, practically guaranteeing the validity of any detections of variability. This project has shown that such unquestionable detections are by no means common. However, as the data base has now grown to cover more than a decade in time, some patterns of long-term variability are beginning to appear, even though they may not have been previously recognizable at the $3 \sigma$ level. What follows is a commentary on the behavior of each individual program star, illustrated with q-u plots and graphs of intrinsic polarization as a function of time in all 5 filters over the entire duration of the monitoring program.

In each q-u plot the data points are filled circles, the mean is a cross drawn to the size of the average error of a single measurement, the standard deviation is represented by a dotted ellipse centered on the mean, and three times the average error of a single measurement is represented 
by a solid ellipse centered on the mean. For each star there is one additional q-u plot showing the mean value for each filter. Note that for all the program stars except $48 \mathrm{Lib}$ it is possible to fit the 5 single-filter data points with a straight line which passes close to the origin. This implies that either the interstellar component of the polarization is small or its position angle is nearly the same as that of the intrinsic component. In either case, the straight line fit gives a good approximation to the position angle of the intrinsic polarization. Any elongation of the distribution of data points along that general direction in the single-filter $\mathrm{q}-\mathrm{u}$ plots is good evidence for intrinsic polarization that is variable in degree but constant in position angle, as is commonly expected for polarization caused by electron scaterring in an equatorially flattened axisymmetric disk. This technique makes it possible to identify variable intrinsic polarization even when it is too small to meet the $3 \sigma$ test.

The graphs of polarization degree and position angle as a function of time for each filter show the intrinsic polarization, calculated by vectorially subtracting the interstellar component determined by Poeckert, Bastien, \& Landstreet (1979) or by McLean \& Brown (1978), using a Serkowski law of the form $p_{I S i}=p_{\max } \exp \left[-1.15 \ln ^{2}\left(\lambda_{\max } / \lambda_{i}\right)\right]$ with parameters as summarized in Table 17.

\subsection{Gamma Cas}

Gamma Cas provides a good example of how the mean values of the polarization in 5 filters can sometimes be nearly collinear in the q-u plane, so that a straight line fit can give a good approximation to the position angle of the intrinsic polarization (see Figure 1, lower right panel). The individual filter plots all show some evidence for elongation of the data point patterns along this direction (especially in $U$ and $R$ ), which is good evidence that there is some real low-level variability. Figure 2, however, shows only slight changes from year to year. The polarization of $\gamma$

Cas, and therefore the state of its circumstellar envelope, appears to have been mostly stable since this monitoring program began.

\subsection{Phi Per}

The polarization of $\phi$ Per has been almost certainly variable from year to year, as may be seen in Figure 3, where the data patterns are clearly elongated along the direction indicated by a straight line fit to the filter means. Some sinusoidal tendencies can be seen in Figure 4, especially in the $R$ bandpass. Periodogram analysis suggests a period on the order of 11 to 12 years. If the circumstellar disk is tilted with respect to the binary orbital plane, as advanced by Clarke \& Bjorkman (1998), it might be expected to precess with a similar period. However, the almost perfectly constant position angle of the polarization argues against this explanation for the periodic polarization. 


\subsection{Per}

With $v \sin i=200 \mathrm{~km} \mathrm{~s}^{-1}, 48$ Per is probably viewed at a relatively low inclination of its rotation axis to the line of sight. This is consistent with its relatively small polarization, even though it is a strong $\mathrm{H} \alpha$ emitter. It is interesting to see from Figure 6 that the position angle shows stronger variability than the degree of polarization, including a hint of a $4-5$ year cycle in the $B$ filter. A precessing bar-shaped nonuniformity embedded in the disk would be expected to generate this kind of variability. The absence of a preferred direction in the q-u plots (Figure 5) lends further strength to this interpretation.

\subsection{Zeta Tau}

Zeta Tau is one of the most highly polarized and strongly variable of all the program stars. A look at Figure 8 shows a slow and steady rise in the degree of polarization with occasional mild outbursts or local maxima, while the position angle remains constant. The filter averages in Figure 7 are very nearly collinear, and the individual filter plots clearly show alignment in that direction.

Work is in progress on a possible correlation between continuum polarization and $V / R$ variations of the $\mathrm{H} \alpha$ emission line profile of $\zeta$ Tau as a test of the theory of "one-armed" density perturbations of the circumstellar disk (Okazaki 1997) and their effects on the polarization. Hopefully the results will place some constraints on the nature of Be disks and the processes leading to their formation.

\section{5. $48 \mathrm{Lib}$}

In Figure 9 the intrinsic position angle of the polarization of 48 Lib is poorly determined because the interstellar component is large and has a very different position angle than the intrinsic component. In Figure 10 the interstellar polarization has been removed, resulting in a better straight line fit that passes acceptably close to the origin to approximate the intrinsic position angle. This angle is indeed favored by the elongations of the q-u data sets in the individual filters.

Figure 11 shows that the degree of intrinsic polarization is nearly cyclic with a period on the order of 4-5 years, although somewhat noisy due to short-term variations. As in the case of $\zeta$ Tau there is a correlation between the polarization period and that of $V / R$ in the $\mathrm{H} \alpha$ emission line, and it is currently being pursued in the same context. 


\subsection{Chi Oph}

Chi Oph has the lowest $v \sin i$ of all the program stars: $140 \mathrm{~km} \mathrm{~s}^{-1}$. It also has one of the least degrees of intrinsic polarization, as would be expected if its rotation axis is only slightly inclined to the line of sight. In Figure 12 the fit of the intrinsic position angle line is somewhat weak, and the individual filter plots have only a slight tendency for alignment.

The graphs of Figure 13 show how small the intrinsic polarization is and how poorly determined the position angle is as a result. Apart from an interesting low-amplitude ripple in the degree of polarization, there is little evidence for any significant variability.

\subsection{Pi Aqr}

Figure 14 shows what $>3 \sigma$ variable polarization of a Be star should look like in the q-u plane. The degree of intrinsic polarization of $\pi$ Aqr has changed more by far than that of any other star on the program list. The strong alignment evident in the $\mathrm{q}-\mathrm{u}$ plots indicates that the position angle is very stable. This is also demonstrated by the position angle graphs of Figure 15 .

When monitoring began in 1985, $\pi$ Aqr had nearly the largest (if not the largest) polarization of any Be star in the sky. Now, 13 years later, that polarization has almost completely disappeared. If we could explain exactly what happened, we would be close to understanding the Be phenomenon itself. Did a dynamic disk lose a source of continuous replenishment? If there were such a source, what might have turned it off? Was the disk a static structure? If so, what prompted it to dissipate? Were there any changes in the underlying star? There are still far more questions than answers.

\subsection{Omicron And}

Since 1986 the polarization of o And has been increasing almost uniformly except for two or three minor outbursts and dropouts. The relatively small degree of polarization makes the position angle difficult to determine (see Figure 16), but Figure 17 shows good definition of the intrinsic position angle based on fitting to the filter means. The polarization is well-behaved in the single filter q-u plots, which show that there is real variability by their strong alignment to the intrinsic position angle. This gives a good record of the gradual buildup of a polarizing Be envelope or disk.

I am very grateful to Michel Breger and Santiago Tapia for introducing me to the basics of

astronomical polarimetry. I also thank Paul Krueger for his skillful machine work in transforming AnyPol from line drawings on paper into the reality of metal. Jon Bjorkman's constructive suggestions as referee helped to clarify this presentation in many ways. 


\section{REFERENCES}

Breger, M. 1979, ApJ, 233, 97

Clarke, D., \& Bjorkman, K. S. 1998, A\&A, 331, 1059

Clarke, D., \& Stewart, B. G. 1986, Vistas Astron., 29, 27

Frecker J. E., \& Serkowski K. 1976, Appl. Optics 15, 605

Hoffleit, D., \& Jaschek, C. 1982, The Bright Star Catalog, 4th Revised Ed. (New Haven, Yale University Observatory)

Hsu, J. C., \& Breger, M. 1982, ApJ, 262, 732

McDavid, D. 1994, PASP, 106, 949

McLean, I. S., \& Brown, J. D. 1978, A\&A, 69, 291

Okazaki, A. T. 1997, A\&A, 318, 548

Poeckert, R., Bastien, P., and Landstreet, J. D. 1979, AJ, 84, 812

Quirrenbach, A., Bjorkman, K. S., Bjorkman, J. E., Hummel, C. A., Buscher, D. F., Armstrong, J. T., Mozurkewich, D., Elias, N. M., II, \& Babler, B. L. 1997, ApJ, 479, 477

Serkowski, K. 1974, in Planets, Stars, and Nebulae Studied with Photopolarimetry, ed. T. Gehrels (Tucson, University of Arizona), p. 135

Slettebak, A. 1982, ApJS, 50, 55

Slettebak, A. 1988, PASP, 100, 770

Wood, K., Bjorkman, K. S., \& Bjorkman, J. E. 1997, ApJ, 477, 926 
Fig.1.-Normalized Stokes parameter plots of the polarization of $\gamma$ Cas (see text for explanation of the symbols).

Fig.2.-Degree and position angle of the intrinsic polarization of $\gamma$ Cas.

Fig.3.-Normalized Stokes parameter plots of the polarization of $\phi$ Per (see text for explanation of the symbols).

Fig.4.-Degree and position angle of the intrinsic polarization of $\phi$ Per.

Fig.5.-Normalized Stokes parameter plots of the polarization of 48 Per (see text for explanation of the symbols).

Fig.6.-Degree and position angle of the intrinsic polarization of 48 Per.

Fig.7.-Normalized Stokes parameter plots of the polarization of $\zeta$ Tau (see text for explanation of the symbols).

Fig.8.-Degree and position angle of the intrinsic polarization of $\zeta$ Tau.

Fig.9.-Normalized Stokes parameter plots of the polarization of 48 Lib (see text for explanation of the symbols).

Fig.10.-Normalized Stokes parameter plots of the intrinsic polarization of $48 \mathrm{Lib}$ (see Subsection 5.5 for comment).

Fig.11.-Degree and position angle of the intrinsic polarization of 48 Lib.

Fig.12.-Normalized Stokes parameter plots of the polarization of $\chi$ Oph (see text for explanation of the symbols).

Fig.13.-Degree and position angle of the intrinsic polarization of $\chi$ Oph.

Fig.14.-Normalized Stokes parameter plots of the polarization of $\pi$ Aqr (see text for explanation of the symbols).

Fig.15.-Degree and position angle of the intrinsic polarization of $\pi$ Aqr.

Fig.16.-Normalized Stokes parameter plots of the polarization of o And (see text for explanation of the symbols).

Fig.17.-Degree and position angle of the intrinsic polarization of o And. 
Table 1. Program Be Stars

\begin{tabular}{lrrlll}
\hline \hline Name & HD & HR & V & Spectral Type & $\begin{array}{c}v \sin i \\
\left(\mathrm{~km} \mathrm{~s}^{-1}\right)\end{array}$ \\
\hline$\gamma$ Cas & 5394 & 264 & 2.47 & B0.5 IVe & 230 \\
$\phi$ Per & 10516 & 496 & 4.07 & B1.5 (V:) e-shell & 400 \\
48 Per & 25940 & 1273 & 4.04 & B4 Ve & 200 \\
$\zeta$ Tau & 37202 & 1910 & 3.00 & B1 IVe-shell & 220 \\
48 Lib & 142983 & 5941 & 4.88 & B3:IV:e-shell & 400 \\
$\chi$ Oph & 148184 & 6118 & 4.42 & B1.5 Ve & 140 \\
$\pi$ Aqr & 212571 & 8539 & 4.66 & B1 III-IVe & 300 \\
$\circ$ And & 217675 & 8762 & 3.62 & B6 III & 260 \\
\hline
\end{tabular}


Table 2. Filter System Parameters

\begin{tabular}{ccc}
\hline \hline Filter & $\begin{array}{c}\text { Effective Wavelength } \\
(\AA)\end{array}$ & $\begin{array}{c}\text { Bandpass (fwhm) } \\
(\AA)\end{array}$ \\
\hline U & 3650 & 700 \\
B & 4400 & 1000 \\
V & 5500 & 900 \\
R & 6400 & 1500 \\
I & 7900 & 1500 \\
\hline
\end{tabular}


Table 3. 2H Cam

\begin{tabular}{|c|c|c|c|c|c|}
\hline $\begin{array}{c}\text { Date }(\mathrm{Mo} / \mathrm{Yr}) \\
\text { Filter }(n)\end{array}$ & $\begin{array}{c}q / d q \\
(\%)\end{array}$ & $\begin{array}{c}u / d u \\
(\%)\end{array}$ & $\begin{array}{c}p / d p \\
(\%)\end{array}$ & $\begin{array}{c}\theta / d \theta \\
\left({ }^{\circ}\right)\end{array}$ & $\begin{array}{l}d p i / d \theta i \\
\left.(\%) \quad{ }^{\circ}\right)\end{array}$ \\
\hline \multicolumn{6}{|l|}{$12 / 94$} \\
\hline $\mathrm{U}(4)$ & $-1.82 / 0.07$ & $-2.33 / 0.06$ & $2.96 / 0.02$ & $116.0 / 0.8$ & $0.09 / 1.3$ \\
\hline$B(4)$ & $-2.06 / 0.03$ & $-2.58 / 0.09$ & $3.30 / 0.08$ & $115.7 / 0.5$ & $0.05 / 0.8$ \\
\hline $\mathrm{V}(4)$ & $-2.07 / 0.08$ & $-2.69 / 0.11$ & $3.40 / 0.10$ & $116.2 / 0.8$ & $0.05 / 0.8$ \\
\hline$R(4)$ & $-2.00 / 0.07$ & $-2.61 / 0.11$ & $3.29 / 0.07$ & $116.3 / 1.0$ & $0.04 / 0.7$ \\
\hline $\mathrm{I}(4)$ & $-1.82 / 0.09$ & $-2.24 / 0.02$ & $2.88 / 0.06$ & $115.5 / 0.7$ & $0.08 / 0.7$ \\
\hline \multicolumn{6}{|l|}{ 01/96 } \\
\hline $\mathrm{U}(3)$ & $-1.94 / 0.01$ & $-2.43 / 0.08$ & $3.11 / 0.05$ & $115.7 / 0.6$ & $0.10 / 1.0$ \\
\hline $\mathrm{B}(3)$ & $-1.97 / 0.11$ & $-2.67 / 0.04$ & $3.32 / 0.10$ & $116.8 / 0.6$ & $0.08 / 0.7$ \\
\hline $\mathrm{V}(3)$ & $-2.06 / 0.09$ & $-2.75 / 0.05$ & $3.44 / 0.09$ & $116.6 / 0.4$ & $0.04 / 0.5$ \\
\hline$R(3)$ & $-1.98 / 0.06$ & $-2.70 / 0.08$ & $3.35 / 0.10$ & $116.9 / 0.3$ & $0.06 / 0.3$ \\
\hline I (3) & $-1.79 / 0.10$ & $-2.43 / 0.04$ & $3.02 / 0.09$ & $116.8 / 0.6$ & $0.11 / 0.9$ \\
\hline \multicolumn{6}{|l|}{$12 / 96$} \\
\hline $\mathrm{U}(3)$ & $-1.73 / 0.02$ & $-2.44 / 0.02$ & $3.00 / 0.02$ & $117.4 / 0.2$ & $0.06 / 0.9$ \\
\hline$B(3)$ & $-1.86 / 0.10$ & $-2.72 / 0.03$ & $3.29 / 0.05$ & $117.8 / 0.8$ & $0.07 / 0.3$ \\
\hline $\mathrm{V}(3)$ & $-2.03 / 0.06$ & $-2.78 / 0.05$ & $3.45 / 0.01$ & $117.0 / 0.6$ & $0.04 / 0.5$ \\
\hline$R(3)$ & $-1.98 / 0.05$ & $-2.66 / 0.03$ & $3.32 / 0.01$ & $116.7 / 0.5$ & $0.05 / 0.4$ \\
\hline $\mathrm{I}(3)$ & $-1.80 / 0.01$ & $-2.41 / 0.08$ & $3.01 / 0.07$ & $116.6 / 0.5$ & $0.10 / 0.7$ \\
\hline \multicolumn{6}{|l|}{$12 / 97$} \\
\hline $\mathrm{U}(5)$ & $-1.81 / 0.06$ & $-2.46 / 0.02$ & $3.06 / 0.04$ & $116.8 / 0.5$ & $0.12 / 1.4$ \\
\hline$B(5)$ & $-1.93 / 0.04$ & $-2.62 / 0.03$ & $3.26 / 0.03$ & $116.8 / 0.4$ & $0.06 / 0.6$ \\
\hline $\mathrm{V}(5)$ & $-2.02 / 0.02$ & $-2.78 / 0.05$ & $3.44 / 0.04$ & $117.0 / 0.4$ & $0.10 / 0.5$ \\
\hline$R(5)$ & $-1.95 / 0.04$ & $-2.67 / 0.04$ & $3.31 / 0.04$ & $116.9 / 0.4$ & $0.05 / 0.7$ \\
\hline$I(5)$ & $-1.74 / 0.08$ & $-2.38 / 0.10$ & $2.95 / 0.09$ & $116.9 / 1.0$ & $0.08 / 0.8$ \\
\hline
\end{tabular}


Table 4. o Sco

\begin{tabular}{|c|c|c|c|c|c|}
\hline $\begin{array}{c}\operatorname{Date}(\mathrm{Mo} / \mathrm{Yr}) \\
\text { Filter }(n)\end{array}$ & $\begin{array}{c}q / d q \\
(\%)\end{array}$ & $\begin{array}{c}u / d u \\
(\%)\end{array}$ & $\begin{array}{l}p / d p \\
(\%)\end{array}$ & $\begin{array}{c}\theta / d \theta \\
\left({ }^{\circ}\right)\end{array}$ & $\begin{array}{l}d p i / d \theta i \\
\left.(\%) \quad{ }^{\circ}\right)\end{array}$ \\
\hline \multicolumn{6}{|l|}{ 06/95 } \\
\hline $\mathrm{U}(4)$ & $1.25 / 0.28$ & $2.40 / 0.17$ & $2.73 / 0.18$ & $31.2 / 2.8$ & $0.17 / 3.2$ \\
\hline$B(4)$ & $1.58 / 0.13$ & $3.03 / 0.06$ & $3.42 / 0.11$ & $31.3 / 0.8$ & $0.09 / 1.1$ \\
\hline $\mathrm{V}(4)$ & $1.96 / 0.08$ & $3.72 / 0.10$ & $4.21 / 0.11$ & $31.2 / 0.4$ & $0.11 / 1.0$ \\
\hline$R(4)$ & $2.05 / 0.05$ & $3.94 / 0.06$ & $4.44 / 0.08$ & $31.3 / 0.2$ & $0.11 / 0.7$ \\
\hline $\mathrm{I}(4)$ & $1.93 / 0.03$ & $3.85 / 0.08$ & $4.31 / 0.07$ & $31.7 / 0.3$ & $0.19 / 1.2$ \\
\hline \multicolumn{6}{|l|}{$06 / 96$} \\
\hline $\mathrm{U}(4)$ & $0.93 / 0.12$ & $2.36 / 0.38$ & $2.54 / 0.39$ & $34.3 / 0.8$ & $0.19 / 2.1$ \\
\hline$B(4)$ & $1.37 / 0.05$ & $3.14 / 0.08$ & $3.43 / 0.10$ & $33.2 / 0.3$ & $0.06 / 0.8$ \\
\hline $\mathrm{V}(4)$ & $1.68 / 0.10$ & $3.81 / 0.05$ & $4.17 / 0.01$ & $33.1 / 0.8$ & $0.10 / 0.9$ \\
\hline$R(4)$ & $1.76 / 0.08$ & $4.07 / 0.07$ & $4.44 / 0.07$ & $33.3 / 0.5$ & $0.09 / 0.8$ \\
\hline$I(4)$ & $1.70 / 0.08$ & $3.98 / 0.03$ & $4.33 / 0.04$ & $33.4 / 0.5$ & $0.06 / 0.8$ \\
\hline \multicolumn{6}{|l|}{$07 / 97$} \\
\hline $\mathrm{U}(5)$ & $1.05 / 0.16$ & $2.34 / 0.20$ & $2.58 / 0.23$ & $33.0 / 1.5$ & $0.24 / 2.5$ \\
\hline$B(5)$ & $1.34 / 0.05$ & $3.14 / 0.04$ & $3.41 / 0.05$ & $33.5 / 0.4$ & $0.06 / 0.5$ \\
\hline $\mathrm{V}(5)$ & $1.57 / 0.12$ & $3.80 / 0.10$ & $4.11 / 0.12$ & $33.8 / 0.7$ & $0.09 / 0.7$ \\
\hline$R(5)$ & $1.68 / 0.10$ & $4.03 / 0.08$ & $4.36 / 0.07$ & $33.7 / 0.7$ & $0.05 / 0.5$ \\
\hline$I(5)$ & $1.56 / 0.13$ & $4.06 / 0.05$ & $4.35 / 0.07$ & $34.5 / 0.8$ & $0.15 / 0.9$ \\
\hline \multicolumn{6}{|l|}{$07 / 98$} \\
\hline $\mathrm{U}(4)$ & $1.00 / 0.15$ & $2.29 / 0.17$ & $2.51 / 0.18$ & $33.3 / 1.7$ & $0.15 / 3.1$ \\
\hline$B(4)$ & $1.37 / 0.07$ & $3.06 / 0.05$ & $3.35 / 0.06$ & $33.0 / 0.5$ & $0.09 / 0.6$ \\
\hline $\mathrm{V}(4)$ & $1.72 / 0.02$ & $3.73 / 0.08$ & $4.11 / 0.08$ & $32.6 / 0.2$ & $0.09 / 0.6$ \\
\hline$R(4)$ & $1.82 / 0.07$ & $3.97 / 0.07$ & $4.37 / 0.08$ & $32.7 / 0.4$ & $0.11 / 0.6$ \\
\hline $\mathrm{I}(4)$ & $1.75 / 0.06$ & $3.85 / 0.03$ & $4.23 / 0.03$ & $32.8 / 0.4$ & $0.15 / 1.0$ \\
\hline
\end{tabular}


Table 5. $\quad \gamma$ Cas

\begin{tabular}{|c|c|c|c|c|c|}
\hline $\begin{array}{c}\text { Date }(\mathrm{Mo} / \mathrm{Yr}) \\
\text { Filter }(n)\end{array}$ & $\begin{array}{c}q / d q \\
(\%)\end{array}$ & $\begin{array}{c}u / d u \\
(\%)\end{array}$ & $\begin{array}{c}p / d p \\
(\%)\end{array}$ & $\begin{array}{c}\theta / d \theta \\
\left({ }^{\circ}\right)\end{array}$ & $\begin{array}{l}d p i / d \theta i \\
(\%) \quad\left({ }^{\circ}\right)\end{array}$ \\
\hline \multicolumn{6}{|l|}{$12 / 94$} \\
\hline $\mathrm{U}(4)$ & $-0.43 / 0.08$ & $-0.24 / 0.08$ & $0.50 / 0.10$ & $103.9 / 3.1$ & $0.07 / 2.5$ \\
\hline$B(4)$ & $-0.66 / 0.05$ & $-0.42 / 0.02$ & $0.79 / 0.04$ & $106.1 / 1.4$ & $0.05 / 1.7$ \\
\hline $\mathrm{V}(4)$ & $-0.58 / 0.04$ & $-0.35 / 0.03$ & $0.69 / 0.02$ & $105.7 / 2.1$ & $0.05 / 1.8$ \\
\hline $\mathrm{R}(4)$ & $-0.62 / 0.06$ & $-0.32 / 0.03$ & $0.70 / 0.05$ & $103.8 / 2.2$ & $0.05 / 1.5$ \\
\hline $\mathrm{I}(4)$ & $-0.54 / 0.05$ & $-0.22 / 0.04$ & $0.59 / 0.05$ & $100.8 / 1.4$ & $0.06 / 3.6$ \\
\hline \multicolumn{6}{|l|}{$01 / 96$} \\
\hline $\mathrm{U}(3)$ & $-0.56 / 0.08$ & $-0.28 / 0.04$ & $0.63 / 0.08$ & $103.5 / 1.8$ & $0.08 / 2.1$ \\
\hline$B(3)$ & $-0.70 / 0.05$ & $-0.50 / 0.12$ & $0.87 / 0.06$ & $107.7 / 3.9$ & $0.03 / 2.5$ \\
\hline $\mathrm{V}(3)$ & $-0.59 / 0.03$ & $-0.40 / 0.05$ & $0.72 / 0.01$ & $107.0 / 2.2$ & $0.05 / 1.5$ \\
\hline$R(3)$ & $-0.60 / 0.08$ & $-0.31 / 0.09$ & $0.68 / 0.08$ & $103.7 / 3.8$ & $0.06 / 2.1$ \\
\hline$I(3)$ & $-0.41 / 0.04$ & $-0.25 / 0.03$ & $0.49 / 0.05$ & $106.4 / 1.4$ & $0.09 / 3.5$ \\
\hline \multicolumn{6}{|l|}{$12 / 96$} \\
\hline $\mathrm{U}(5)$ & $-0.38 / 0.05$ & $-0.30 / 0.08$ & $0.49 / 0.07$ & $109.3 / 3.1$ & $0.07 / 3.7$ \\
\hline$B(5)$ & $-0.61 / 0.06$ & $-0.42 / 0.08$ & $0.74 / 0.06$ & $107.3 / 3.1$ & $0.07 / 2.9$ \\
\hline $\mathrm{V}(5)$ & $-0.56 / 0.08$ & $-0.33 / 0.09$ & $0.66 / 0.09$ & $105.3 / 3.1$ & $0.06 / 2.6$ \\
\hline$R(5)$ & $-0.55 / 0.08$ & $-0.28 / 0.08$ & $0.62 / 0.07$ & $103.2 / 3.8$ & $0.10 / 3.2$ \\
\hline I (5) & $-0.46 / 0.06$ & $-0.24 / 0.06$ & $0.52 / 0.07$ & $103.2 / 3.0$ & $0.09 / 3.2$ \\
\hline \multicolumn{6}{|l|}{$12 / 97$} \\
\hline $\mathrm{U}(6)$ & $-0.50 / 0.08$ & $-0.31 / 0.03$ & $0.60 / 0.08$ & $106.2 / 1.6$ & $0.08 / 4.9$ \\
\hline$B(6)$ & $-0.64 / 0.04$ & $-0.39 / 0.09$ & $0.76 / 0.08$ & $105.6 / 2.5$ & $0.11 / 2.9$ \\
\hline $\mathrm{V}(6)$ & $-0.58 / 0.06$ & $-0.40 / 0.06$ & $0.70 / 0.08$ & $107.2 / 1.2$ & $0.08 / 2.3$ \\
\hline$R(6)$ & $-0.58 / 0.06$ & $-0.27 / 0.08$ & $0.65 / 0.04$ & $102.4 / 4.0$ & $0.11 / 3.4$ \\
\hline$I(6)$ & $-0.45 / 0.05$ & $-0.20 / 0.05$ & $0.50 / 0.05$ & $102.2 / 2.6$ & $0.06 / 6.8$ \\
\hline
\end{tabular}


Table 6. $\phi$ Per

\begin{tabular}{|c|c|c|c|c|c|}
\hline $\begin{array}{c}\text { Date (Mo/Yr) } \\
\text { Filter }(n)\end{array}$ & $\begin{array}{c}q / d q \\
(\%)\end{array}$ & $\begin{array}{c}u / d u \\
(\%)\end{array}$ & $\begin{array}{c}p / d p \\
(\%)\end{array}$ & $\begin{array}{c}\theta / d \theta \\
\left({ }^{\circ}\right)\end{array}$ & $\begin{array}{l}d p i / d \theta i \\
\left.(\%) \quad{ }^{\circ}\right)\end{array}$ \\
\hline \multicolumn{6}{|l|}{$12 / 94$} \\
\hline $\mathrm{U}(4)$ & $0.25 / 0.06$ & $0.85 / 0.10$ & $0.89 / 0.10$ & $36.9 / 2.2$ & $0.08 / 2.0$ \\
\hline$B(4)$ & $0.46 / 0.11$ & $1.09 / 0.08$ & $1.19 / 0.08$ & $33.6 / 2.5$ & $0.04 / 1.7$ \\
\hline $\mathrm{V}(4)$ & $0.34 / 0.08$ & $0.99 / 0.08$ & $1.05 / 0.09$ & $35.5 / 1.8$ & $0.05 / 1.5$ \\
\hline$R(4)$ & $0.24 / 0.07$ & $0.90 / 0.08$ & $0.94 / 0.07$ & $37.5 / 2.2$ & $0.06 / 2.6$ \\
\hline$I(4)$ & $0.10 / 0.08$ & $0.72 / 0.03$ & $0.73 / 0.03$ & $41.0 / 3.0$ & $0.10 / 2.4$ \\
\hline \multicolumn{6}{|l|}{$01 / 96$} \\
\hline $\mathrm{U}(3)$ & $0.04 / 0.08$ & $0.85 / 0.11$ & $0.86 / 0.11$ & $43.7 / 2.5$ & $0.07 / 3.8$ \\
\hline$B(3)$ & $0.41 / 0.06$ & $1.23 / 0.06$ & $1.30 / 0.07$ & $35.8 / 1.2$ & $0.06 / 1.2$ \\
\hline $\mathrm{V}(3)$ & $0.37 / 0.07$ & $1.10 / 0.08$ & $1.16 / 0.09$ & $35.8 / 1.4$ & $0.07 / 1.1$ \\
\hline$R(3)$ & $0.25 / 0.03$ & $0.94 / 0.09$ & $0.97 / 0.08$ & $37.5 / 1.2$ & $0.06 / 1.4$ \\
\hline $\mathrm{I}(3)$ & $0.22 / 0.03$ & $0.69 / 0.08$ & $0.73 / 0.09$ & $36.5 / 0.4$ & $0.11 / 2.6$ \\
\hline \multicolumn{6}{|l|}{$12 / 96$} \\
\hline $\mathrm{U}(5)$ & $0.18 / 0.05$ & $0.81 / 0.12$ & $0.83 / 0.12$ & $38.5 / 1.6$ & $0.10 / 2.1$ \\
\hline$B(5)$ & $0.50 / 0.04$ & $1.28 / 0.10$ & $1.37 / 0.08$ & $34.3 / 1.5$ & $0.05 / 1.4$ \\
\hline $\mathrm{V}(5)$ & $0.39 / 0.06$ & $1.12 / 0.10$ & $1.19 / 0.10$ & $35.4 / 1.3$ & $0.07 / 1.6$ \\
\hline$R(5)$ & $0.25 / 0.05$ & $1.01 / 0.10$ & $1.05 / 0.10$ & $37.8 / 1.5$ & $0.05 / 2.6$ \\
\hline$I(5)$ & $0.19 / 0.07$ & $0.81 / 0.17$ & $0.84 / 0.17$ & $38.1 / 2.3$ & $0.06 / 3.2$ \\
\hline \multicolumn{6}{|l|}{$12 / 97$} \\
\hline $\mathrm{U}(6)$ & $0.15 / 0.07$ & $0.83 / 0.08$ & $0.85 / 0.08$ & $39.7 / 2.1$ & $0.07 / 3.8$ \\
\hline$B(6)$ & $0.52 / 0.01$ & $1.34 / 0.06$ & $1.44 / 0.06$ & $34.4 / 0.4$ & $0.05 / 1.3$ \\
\hline $\mathrm{V}(6)$ & $0.44 / 0.04$ & $1.21 / 0.04$ & $1.29 / 0.05$ & $35.0 / 0.8$ & $0.07 / 2.3$ \\
\hline$R(6)$ & $0.33 / 0.02$ & $1.09 / 0.08$ & $1.14 / 0.07$ & $36.6 / 0.7$ & $0.05 / 1.8$ \\
\hline$I(6)$ & $0.29 / 0.05$ & $0.95 / 0.08$ & $1.00 / 0.08$ & $36.5 / 1.7$ & $0.09 / 3.2$ \\
\hline
\end{tabular}


Table 7. 48 Per

\begin{tabular}{|c|c|c|c|c|c|}
\hline $\begin{array}{c}\text { Date }(\mathrm{Mo} / \mathrm{Yr}) \\
\text { Filter }(n)\end{array}$ & $\begin{array}{c}q / d q \\
(\%)\end{array}$ & $\begin{array}{c}u / d u \\
(\%)\end{array}$ & $\begin{array}{l}p / d p \\
(\%)\end{array}$ & $\begin{array}{c}\theta / d \theta \\
\left({ }^{\circ}\right)\end{array}$ & $\begin{array}{l}d p i / d \theta i \\
(\%) \quad\left({ }^{\circ}\right)\end{array}$ \\
\hline \multicolumn{6}{|l|}{$12 / 94$} \\
\hline $\mathrm{U}(4)$ & $0.77 / 0.05$ & $-0.21 / 0.06$ & $0.81 / 0.04$ & $172.3 / 2.7$ & $0.11 / 2.2$ \\
\hline$B(4)$ & $0.76 / 0.06$ & $-0.28 / 0.05$ & $0.81 / 0.04$ & $169.7 / 2.3$ & $0.05 / 1.8$ \\
\hline $\mathrm{V}(4)$ & $0.91 / 0.07$ & $-0.34 / 0.07$ & $0.97 / 0.05$ & $169.9 / 2.4$ & $0.09 / 1.8$ \\
\hline$R(4)$ & $0.85 / 0.05$ & $-0.29 / 0.03$ & $0.90 / 0.05$ & $170.6 / 1.2$ & $0.07 / 1.3$ \\
\hline $\mathrm{I}(4)$ & $0.72 / 0.05$ & $-0.23 / 0.06$ & $0.76 / 0.05$ & $171.2 / 2.2$ & $0.06 / 2.8$ \\
\hline \multicolumn{6}{|l|}{$01 / 96$} \\
\hline $\mathrm{U}(3)$ & $0.61 / 0.06$ & $-0.14 / 0.04$ & $0.63 / 0.07$ & $173.3 / 2.1$ & $0.09 / 3.3$ \\
\hline$B(3)$ & $0.82 / 0.03$ & $-0.20 / 0.06$ & $0.85 / 0.03$ & $173.2 / 1.8$ & $0.03 / 1.8$ \\
\hline $\mathrm{V}(3)$ & $0.89 / 0.05$ & $-0.20 / 0.06$ & $0.91 / 0.04$ & $173.6 / 2.1$ & $0.05 / 3.0$ \\
\hline$R(3)$ & $0.92 / 0.05$ & $-0.24 / 0.04$ & $0.96 / 0.05$ & $172.6 / 1.4$ & $0.07 / 0.8$ \\
\hline$I(3)$ & $0.81 / 0.04$ & $-0.17 / 0.05$ & $0.83 / 0.04$ & $174.1 / 1.8$ & $0.08 / 1.3$ \\
\hline \multicolumn{6}{|l|}{$12 / 96$} \\
\hline $\mathrm{U}(4)$ & $0.83 / 0.07$ & $-0.24 / 0.08$ & $0.87 / 0.05$ & $171.6 / 3.3$ & $0.08 / 2.0$ \\
\hline$B(4)$ & $0.90 / 0.02$ & $-0.25 / 0.04$ & $0.94 / 0.02$ & $172.1 / 1.2$ & $0.07 / 1.4$ \\
\hline $\mathrm{V}(4)$ & $0.96 / 0.07$ & $-0.24 / 0.06$ & $0.99 / 0.08$ & $172.9 / 1.7$ & $0.06 / 2.4$ \\
\hline$R(4)$ & $0.97 / 0.04$ & $-0.21 / 0.09$ & $1.00 / 0.02$ & $173.9 / 2.7$ & $0.07 / 2.0$ \\
\hline $\mathrm{I}(4)$ & $0.85 / 0.09$ & $-0.25 / 0.08$ & $0.90 / 0.08$ & $171.5 / 2.9$ & $0.11 / 4.4$ \\
\hline \multicolumn{6}{|l|}{$12 / 97$} \\
\hline $\mathrm{U}(6)$ & $0.71 / 0.06$ & $-0.23 / 0.07$ & $0.75 / 0.07$ & $170.9 / 2.0$ & $0.08 / 2.9$ \\
\hline$B(6)$ & $0.83 / 0.04$ & $-0.22 / 0.05$ & $0.86 / 0.04$ & $172.5 / 1.3$ & $0.06 / 2.2$ \\
\hline $\mathrm{V}(6)$ & $0.97 / 0.08$ & $-0.23 / 0.03$ & $1.00 / 0.08$ & $173.3 / 0.8$ & $0.08 / 2.0$ \\
\hline$R(6)$ & $0.90 / 0.03$ & $-0.21 / 0.06$ & $0.93 / 0.03$ & $173.5 / 2.0$ & $0.08 / 2.3$ \\
\hline$I(6)$ & $0.88 / 0.12$ & $-0.17 / 0.07$ & $0.90 / 0.12$ & $174.4 / 2.6$ & $0.10 / 2.7$ \\
\hline
\end{tabular}


Table 8. $\quad \zeta$ Tau

\begin{tabular}{|c|c|c|c|c|c|}
\hline $\begin{array}{c}\text { Date }(\operatorname{Mo} / \mathrm{Yr}) \\
\text { Filter }(n)\end{array}$ & $\begin{array}{c}q / d q \\
(\%)\end{array}$ & $\begin{array}{c}u / d u \\
(\%)\end{array}$ & $\begin{array}{c}p / d p \\
(\%)\end{array}$ & $\begin{array}{c}\theta / d \theta \\
\left({ }^{\circ}\right)\end{array}$ & $\begin{array}{l}d p i / d \theta i \\
(\%) \quad\left({ }^{\circ}\right)\end{array}$ \\
\hline \multicolumn{6}{|l|}{$12 / 94$} \\
\hline $\mathrm{U}(4)$ & $0.51 / 0.06$ & $1.12 / 0.06$ & $1.24 / 0.06$ & $32.8 / 1.3$ & $0.07 / 1.2$ \\
\hline$B(4)$ & $0.57 / 0.08$ & $1.31 / 0.08$ & $1.43 / 0.10$ & $33.3 / 1.0$ & $0.04 / 0.9$ \\
\hline $\mathrm{V}(4)$ & $0.63 / 0.06$ & $1.24 / 0.05$ & $1.39 / 0.04$ & $31.5 / 1.6$ & $0.03 / 0.7$ \\
\hline$R(4)$ & $0.49 / 0.01$ & $1.15 / 0.02$ & $1.26 / 0.02$ & $33.4 / 0.3$ & $0.06 / 1.3$ \\
\hline$I(4)$ & $0.31 / 0.06$ & $1.05 / 0.10$ & $1.10 / 0.10$ & $36.7 / 1.6$ & $0.07 / 1.4$ \\
\hline \multicolumn{6}{|l|}{$01 / 96$} \\
\hline $\mathrm{U}(3)$ & $0.44 / 0.04$ & $1.01 / 0.08$ & $1.10 / 0.06$ & $33.2 / 1.5$ & $0.04 / 1.2$ \\
\hline$B(3)$ & $0.65 / 0.01$ & $1.26 / 0.10$ & $1.42 / 0.08$ & $31.3 / 1.0$ & $0.05 / 1.3$ \\
\hline $\mathrm{V}(3)$ & $0.59 / 0.05$ & $1.25 / 0.08$ & $1.38 / 0.08$ & $32.4 / 0.3$ & $0.05 / 1.7$ \\
\hline$R(3)$ & $0.51 / 0.02$ & $1.20 / 0.02$ & $1.30 / 0.02$ & $33.5 / 0.4$ & $0.10 / 1.5$ \\
\hline $\mathrm{I}(3)$ & $0.52 / 0.03$ & $1.06 / 0.10$ & $1.18 / 0.10$ & $31.9 / 0.5$ & $0.06 / 1.6$ \\
\hline \multicolumn{6}{|l|}{$12 / 96$} \\
\hline $\mathrm{U}(4)$ & $0.59 / 0.02$ & $1.04 / 0.04$ & $1.21 / 0.03$ & $30.2 / 0.6$ & $0.08 / 2.9$ \\
\hline $\mathrm{B}(4)$ & $0.64 / 0.04$ & $1.42 / 0.05$ & $1.57 / 0.04$ & $32.8 / 0.8$ & $0.05 / 2.8$ \\
\hline $\mathrm{V}(4)$ & $0.59 / 0.03$ & $1.30 / 0.05$ & $1.44 / 0.03$ & $32.8 / 0.9$ & $0.06 / 2.3$ \\
\hline$R(4)$ & $0.52 / 0.04$ & $1.26 / 0.05$ & $1.36 / 0.04$ & $33.8 / 1.1$ & $0.11 / 1.5$ \\
\hline$I(4)$ & $0.44 / 0.06$ & $1.01 / 0.10$ & $1.11 / 0.11$ & $33.3 / 0.9$ & $0.11 / 2.5$ \\
\hline \multicolumn{6}{|l|}{$12 / 97$} \\
\hline $\mathrm{U}(6)$ & $0.50 / 0.08$ & $1.08 / 0.08$ & $1.20 / 0.06$ & $32.6 / 2.3$ & $0.09 / 2.5$ \\
\hline$B(6)$ & $0.74 / 0.07$ & $1.45 / 0.11$ & $1.63 / 0.09$ & $31.5 / 1.4$ & $0.08 / 2.0$ \\
\hline $\mathrm{V}(6)$ & $0.71 / 0.05$ & $1.37 / 0.12$ & $1.55 / 0.10$ & $31.3 / 1.5$ & $0.10 / 1.8$ \\
\hline$R(6)$ & $0.55 / 0.07$ & $1.30 / 0.11$ & $1.42 / 0.10$ & $33.5 / 1.7$ & $0.07 / 2.0$ \\
\hline$I(6)$ & $0.57 / 0.02$ & $1.12 / 0.12$ & $1.26 / 0.11$ & $31.4 / 1.1$ & $0.07 / 2.5$ \\
\hline
\end{tabular}


Table 9. 48 Lib

\begin{tabular}{|c|c|c|c|c|c|}
\hline $\begin{array}{c}\text { Date }(\mathrm{Mo} / \mathrm{Yr}) \\
\text { Filter }(n)\end{array}$ & $\begin{array}{c}q / d q \\
(\%)\end{array}$ & $\begin{array}{c}u / d u \\
(\%)\end{array}$ & $\begin{array}{c}p / d p \\
(\%)\end{array}$ & $\begin{array}{c}\theta / d \theta \\
\left({ }^{\circ}\right)\end{array}$ & $\begin{array}{l}d p i / d \theta i \\
(\%) \quad\left({ }^{\circ}\right)\end{array}$ \\
\hline \multicolumn{6}{|l|}{$06 / 95$} \\
\hline $\mathrm{U}(4)$ & $-0.40 / 0.12$ & $-0.61 / 0.07$ & $0.74 / 0.10$ & $118.3 / 4.4$ & $0.13 / 5.9$ \\
\hline$B(4)$ & $-0.36 / 0.06$ & $-0.83 / 0.06$ & $0.91 / 0.05$ & $123.3 / 2.2$ & $0.03 / 2.9$ \\
\hline $\mathrm{V}(4)$ & $-0.46 / 0.03$ & $-0.71 / 0.04$ & $0.85 / 0.04$ & $118.7 / 1.4$ & $0.06 / 2.2$ \\
\hline$R(4)$ & $-0.43 / 0.05$ & $-0.60 / 0.04$ & $0.74 / 0.02$ & $117.1 / 2.3$ & $0.07 / 2.1$ \\
\hline$I(4)$ & $-0.51 / 0.06$ & $-0.54 / 0.13$ & $0.76 / 0.10$ & $112.6 / 4.1$ & $0.10 / 4.9$ \\
\hline \multicolumn{6}{|l|}{$06 / 96$} \\
\hline $\mathrm{U}(4)$ & $-0.43 / 0.04$ & $-0.69 / 0.09$ & $0.82 / 0.06$ & $118.9 / 2.8$ & $0.12 / 3.0$ \\
\hline$B(4)$ & $-0.40 / 0.03$ & $-0.87 / 0.06$ & $0.96 / 0.07$ & $122.6 / 0.7$ & $0.07 / 2.6$ \\
\hline $\mathrm{V}(4)$ & $-0.52 / 0.05$ & $-0.73 / 0.08$ & $0.90 / 0.09$ & $117.2 / 1.0$ & $0.06 / 1.9$ \\
\hline$R(4)$ & $-0.55 / 0.07$ & $-0.64 / 0.06$ & $0.86 / 0.07$ & $114.7 / 2.1$ & $0.06 / 2.6$ \\
\hline $\mathrm{I}(4)$ & $-0.52 / 0.02$ & $-0.51 / 0.11$ & $0.74 / 0.08$ & $112.2 / 3.1$ & $0.12 / 5.4$ \\
\hline \multicolumn{6}{|l|}{$07 / 97$} \\
\hline $\mathrm{U}(4)$ & $-0.35 / 0.10$ & $-0.41 / 0.06$ & $0.56 / 0.06$ & $114.8 / 5.0$ & $0.12 / 6.0$ \\
\hline$B(4)$ & $-0.46 / 0.05$ & $-0.72 / 0.08$ & $0.86 / 0.08$ & $118.5 / 1.5$ & $0.09 / 1.6$ \\
\hline $\mathrm{V}(4)$ & $-0.60 / 0.04$ & $-0.65 / 0.05$ & $0.88 / 0.06$ & $113.8 / 1.2$ & $0.11 / 1.9$ \\
\hline$R(4)$ & $-0.57 / 0.09$ & $-0.63 / 0.05$ & $0.85 / 0.09$ & $114.0 / 1.4$ & $0.09 / 2.6$ \\
\hline $\mathrm{I}(4)$ & $-0.67 / 0.11$ & $-0.60 / 0.09$ & $0.91 / 0.12$ & $111.4 / 2.1$ & $0.11 / 5.3$ \\
\hline \multicolumn{6}{|l|}{$07 / 98$} \\
\hline $\mathrm{U}(5)$ & $-0.35 / 0.08$ & $-0.53 / 0.08$ & $0.65 / 0.10$ & $118.5 / 2.3$ & $0.09 / 5.4$ \\
\hline$B(5)$ & $-0.42 / 0.06$ & $-0.78 / 0.08$ & $0.89 / 0.06$ & $120.7 / 2.6$ & $0.04 / 3.9$ \\
\hline $\mathrm{V}(5)$ & $-0.51 / 0.04$ & $-0.71 / 0.08$ & $0.88 / 0.08$ & $117.1 / 1.0$ & $0.07 / 3.0$ \\
\hline$R(5)$ & $-0.53 / 0.02$ & $-0.63 / 0.06$ & $0.83 / 0.04$ & $114.8 / 1.8$ & $0.09 / 2.3$ \\
\hline $\mathrm{I}(5)$ & $-0.42 / 0.03$ & $-0.58 / 0.09$ & $0.74 / 0.06$ & $117.2 / 3.0$ & $0.14 / 6.6$ \\
\hline
\end{tabular}


Table 10. $\chi$ Oph

\begin{tabular}{|c|c|c|c|c|c|}
\hline $\begin{array}{c}\text { Date (Mo/Yr) } \\
\text { Filter }(n)\end{array}$ & $\begin{array}{c}q / d q \\
(\%)\end{array}$ & $\begin{array}{c}u / d u \\
(\%)\end{array}$ & $\begin{array}{c}p / d p \\
(\%)\end{array}$ & $\begin{array}{c}\theta / d \theta \\
\left({ }^{\circ}\right)\end{array}$ & $\begin{array}{l}d p i / d \theta i \\
\left.(\%) \quad{ }^{\circ}\right)\end{array}$ \\
\hline \multicolumn{6}{|l|}{ 06/95 } \\
\hline $\mathrm{U}(4)$ & $0.03 / 0.06$ & $-0.39 / 0.05$ & $0.41 / 0.06$ & $137.6 / 4.9$ & $0.11 / 8.5$ \\
\hline$B(4)$ & $-0.06 / 0.06$ & $-0.52 / 0.08$ & $0.54 / 0.08$ & $132.1 / 2.9$ & $0.06 / 5.0$ \\
\hline $\mathrm{V}(4)$ & $0.00 / 0.05$ & $-0.52 / 0.06$ & $0.53 / 0.06$ & $134.8 / 2.9$ & $0.10 / 5.4$ \\
\hline$R(4)$ & $-0.04 / 0.07$ & $-0.46 / 0.07$ & $0.47 / 0.07$ & $132.7 / 4.4$ & $0.05 / 4.8$ \\
\hline$I(4)$ & $-0.02 / 0.04$ & $-0.43 / 0.06$ & $0.45 / 0.04$ & $136.0 / 3.5$ & $0.15 / 12.1$ \\
\hline \multicolumn{6}{|l|}{ 06/96 } \\
\hline $\mathrm{U}(4)$ & $-0.03 / 0.20$ & $-0.28 / 0.03$ & $0.38 / 0.05$ & $134.9 / 18.7$ & $0.12 / 15.7$ \\
\hline$B(4)$ & $0.00 / 0.08$ & $-0.42 / 0.08$ & $0.44 / 0.07$ & $135.5 / 8.3$ & $0.09 / 9.2$ \\
\hline $\mathrm{V}(4)$ & $0.01 / 0.08$ & $-0.45 / 0.07$ & $0.46 / 0.07$ & $135.8 / 4.9$ & $0.13 / 3.9$ \\
\hline$R(4)$ & $-0.05 / 0.07$ & $-0.47 / 0.06$ & $0.48 / 0.06$ & $131.8 / 3.7$ & $0.14 / 6.0$ \\
\hline $\mathrm{I}(4)$ & $-0.16 / 0.06$ & $-0.41 / 0.05$ & $0.47 / 0.05$ & $126.0 / 5.7$ & $0.10 / 12.4$ \\
\hline \multicolumn{6}{|l|}{$07 / 97$} \\
\hline $\mathrm{U}(4)$ & $0.12 / 0.11$ & $-0.28 / 0.05$ & $0.36 / 0.05$ & $144.7 / 9.8$ & $0.08 / 18.9$ \\
\hline$B(4)$ & $-0.01 / 0.12$ & $-0.45 / 0.05$ & $0.47 / 0.05$ & $135.0 / 7.4$ & $0.08 / 4.6$ \\
\hline $\mathrm{V}(4)$ & $0.00 / 0.19$ & $-0.44 / 0.07$ & $0.48 / 0.07$ & $136.2 / 11.5$ & $0.08 / 5.5$ \\
\hline$R(4)$ & $0.05 / 0.13$ & $-0.48 / 0.07$ & $0.50 / 0.06$ & $138.7 / 7.7$ & $0.07 / 7.6$ \\
\hline$I(4)$ & $-0.04 / 0.11$ & $-0.38 / 0.11$ & $0.41 / 0.10$ & $134.5 / 10.6$ & $0.13 / 6.1$ \\
\hline \multicolumn{6}{|l|}{ 07/98 } \\
\hline $\mathrm{U}(5)$ & $0.03 / 0.13$ & $-0.37 / 0.08$ & $0.40 / 0.10$ & $134.7 / 7.6$ & $0.13 / 10.7$ \\
\hline$B(5)$ & $0.01 / 0.07$ & $-0.47 / 0.05$ & $0.48 / 0.05$ & $135.6 / 4.6$ & $0.12 / 4.8$ \\
\hline $\mathrm{V}(5)$ & $-0.03 / 0.04$ & $-0.47 / 0.06$ & $0.49 / 0.06$ & $133.4 / 2.7$ & $0.10 / 7.9$ \\
\hline$R(5)$ & $0.01 / 0.07$ & $-0.47 / 0.03$ & $0.48 / 0.03$ & $135.6 / 3.6$ & $0.08 / 7.1$ \\
\hline$I(5)$ & $0.01 / 0.14$ & $-0.46 / 0.08$ & $0.50 / 0.07$ & $135.5 / 8.4$ & $0.12 / 8.6$ \\
\hline
\end{tabular}


Table 11. $\pi$ Aqr

\begin{tabular}{|c|c|c|c|c|c|}
\hline $\begin{array}{c}\text { Date (Mo/Yr) } \\
\text { Filter }(n)\end{array}$ & $\begin{array}{c}q / d q \\
(\%)\end{array}$ & $\begin{array}{c}u / d u \\
(\%)\end{array}$ & $\begin{array}{c}p / d p \\
(\%)\end{array}$ & $\begin{array}{c}\theta / d \theta \\
\left({ }^{\circ}\right)\end{array}$ & $\begin{array}{l}d p i / d \theta i \\
\left.(\%) \quad{ }^{\circ}\right)\end{array}$ \\
\hline \multicolumn{6}{|l|}{ 06/95 } \\
\hline $\mathrm{U}(3)$ & $-0.01 / 0.11$ & $-0.44 / 0.15$ & $0.47 / 0.14$ & $131.6 / 7.0$ & $0.13 / 12.1$ \\
\hline$B(3)$ & $-0.08 / 0.07$ & $-0.50 / 0.04$ & $0.51 / 0.04$ & $130.0 / 3.8$ & $0.10 / 5.1$ \\
\hline $\mathrm{V}(3)$ & $-0.06 / 0.05$ & $-0.48 / 0.00$ & $0.49 / 0.01$ & $130.9 / 3.0$ & $0.09 / 4.7$ \\
\hline$R(3)$ & $-0.05 / 0.04$ & $-0.45 / 0.04$ & $0.46 / 0.03$ & $132.0 / 2.5$ & $0.09 / 4.0$ \\
\hline$I(3)$ & $0.04 / 0.03$ & $-0.40 / 0.01$ & $0.42 / 0.02$ & $137.8 / 2.4$ & $0.08 / 9.4$ \\
\hline \multicolumn{6}{|l|}{ 06/96 } \\
\hline $\mathrm{U}(4)$ & $-0.34 / 0.03$ & $-0.32 / 0.05$ & $0.48 / 0.04$ & $112.2 / 2.3$ & $0.10 / 5.2$ \\
\hline$B(4)$ & $-0.38 / 0.04$ & $-0.31 / 0.02$ & $0.50 / 0.02$ & $109.6 / 2.3$ & $0.07 / 5.4$ \\
\hline $\mathrm{V}(4)$ & $-0.41 / 0.11$ & $-0.30 / 0.07$ & $0.52 / 0.06$ & $108.9 / 6.0$ & $0.08 / 3.6$ \\
\hline$R(4)$ & $-0.37 / 0.08$ & $-0.29 / 0.03$ & $0.48 / 0.06$ & $109.5 / 3.8$ & $0.09 / 4.6$ \\
\hline $\mathrm{I}(4)$ & $-0.29 / 0.14$ & $-0.31 / 0.04$ & $0.46 / 0.08$ & $114.2 / 9.5$ & $0.14 / 10.4$ \\
\hline \multicolumn{6}{|l|}{$07 / 97$} \\
\hline $\mathrm{U}(4)$ & $-0.26 / 0.02$ & $-0.29 / 0.05$ & $0.40 / 0.05$ & $113.0 / 2.8$ & $0.10 / 7.8$ \\
\hline$B(4)$ & $-0.36 / 0.02$ & $-0.34 / 0.07$ & $0.50 / 0.06$ & $111.8 / 2.5$ & $0.08 / 3.1$ \\
\hline $\mathrm{V}(4)$ & $-0.40 / 0.04$ & $-0.31 / 0.03$ & $0.51 / 0.03$ & $108.8 / 2.4$ & $0.04 / 2.8$ \\
\hline$R(4)$ & $-0.37 / 0.06$ & $-0.31 / 0.03$ & $0.49 / 0.03$ & $110.1 / 3.2$ & $0.08 / 3.4$ \\
\hline$I(4)$ & $-0.31 / 0.08$ & $-0.26 / 0.08$ & $0.43 / 0.07$ & $110.4 / 7.0$ & $0.07 / 9.6$ \\
\hline \multicolumn{6}{|l|}{ 07/98 } \\
\hline $\mathrm{U}(5)$ & $-0.37 / 0.10$ & $-0.27 / 0.09$ & $0.48 / 0.11$ & $107.6 / 4.9$ & $0.10 / 6.4$ \\
\hline$B(5)$ & $-0.36 / 0.06$ & $-0.32 / 0.03$ & $0.49 / 0.05$ & $110.8 / 2.5$ & $0.05 / 2.4$ \\
\hline $\mathrm{V}(5)$ & $-0.40 / 0.04$ & $-0.30 / 0.05$ & $0.51 / 0.02$ & $108.6 / 3.5$ & $0.08 / 4.5$ \\
\hline$R(5)$ & $-0.41 / 0.06$ & $-0.27 / 0.07$ & $0.50 / 0.08$ & $106.7 / 2.4$ & $0.07 / 4.5$ \\
\hline$I(5)$ & $-0.33 / 0.11$ & $-0.25 / 0.08$ & $0.44 / 0.10$ & $110.8 / 5.6$ & $0.09 / 9.5$ \\
\hline
\end{tabular}


Table 12. o And

\begin{tabular}{|c|c|c|c|c|c|}
\hline $\begin{array}{c}\text { Date }(\mathrm{Mo} / \mathrm{Yr}) \\
\text { Filter }(n)\end{array}$ & $\begin{array}{c}q / d q \\
(\%)\end{array}$ & $\begin{array}{c}u / d u \\
(\%)\end{array}$ & $\begin{array}{c}p / d p \\
(\%)\end{array}$ & $\begin{array}{c}\theta / d \theta \\
\left({ }^{\circ}\right)\end{array}$ & $\begin{array}{l}d p i / d \theta i \\
\left.(\%) \quad{ }^{\circ}\right)\end{array}$ \\
\hline \multicolumn{6}{|l|}{ 06/95 } \\
\hline $\mathrm{U}(3)$ & $-0.48 / 0.02$ & $-0.11 / 0.03$ & $0.50 / 0.01$ & $96.5 / 1.8$ & $0.07 / 5.0$ \\
\hline$B(3)$ & $-0.56 / 0.07$ & $-0.16 / 0.11$ & $0.59 / 0.09$ & $97.8 / 4.4$ & $0.03 / 3.2$ \\
\hline $\mathrm{V}(3)$ & $-0.53 / 0.01$ & $-0.05 / 0.01$ & $0.54 / 0.02$ & $92.6 / 0.7$ & $0.06 / 1.0$ \\
\hline$R(3)$ & $-0.48 / 0.03$ & $-0.03 / 0.02$ & $0.49 / 0.03$ & $91.8 / 1.0$ & $0.04 / 2.9$ \\
\hline $\mathrm{I}(3)$ & $-0.46 / 0.09$ & $-0.06 / 0.07$ & $0.48 / 0.10$ & $93.6 / 3.4$ & $0.05 / 7.6$ \\
\hline \multicolumn{6}{|l|}{ 06/96 } \\
\hline $\mathrm{U}(4)$ & $-0.40 / 0.07$ & $-0.06 / 0.07$ & $0.41 / 0.07$ & $93.8 / 4.7$ & $0.09 / 6.6$ \\
\hline$B(4)$ & $-0.37 / 0.04$ & $-0.05 / 0.06$ & $0.38 / 0.04$ & $93.6 / 3.6$ & $0.08 / 3.6$ \\
\hline $\mathrm{V}(4)$ & $-0.32 / 0.06$ & $-0.07 / 0.07$ & $0.35 / 0.05$ & $96.7 / 5.7$ & $0.05 / 9.2$ \\
\hline$R(4)$ & $-0.29 / 0.07$ & $-0.06 / 0.04$ & $0.31 / 0.06$ & $95.6 / 4.0$ & $0.07 / 9.8$ \\
\hline I (4) & $-0.30 / 0.10$ & $-0.04 / 0.08$ & $0.32 / 0.11$ & $94.3 / 6.0$ & $0.11 / 8.9$ \\
\hline \multicolumn{6}{|l|}{$07 / 97$} \\
\hline $\mathrm{U}(4)$ & $-0.27 / 0.03$ & $0.00 / 0.06$ & $0.28 / 0.03$ & $89.7 / 6.4$ & $0.10 / 7.5$ \\
\hline$B(4)$ & $-0.36 / 0.02$ & $-0.05 / 0.05$ & $0.37 / 0.02$ & $94.1 / 4.6$ & $0.06 / 4.4$ \\
\hline $\mathrm{V}(4)$ & $-0.36 / 0.05$ & $-0.02 / 0.01$ & $0.37 / 0.05$ & $91.0 / 1.1$ & $0.06 / 7.4$ \\
\hline$R(4)$ & $-0.35 / 0.04$ & $-0.04 / 0.02$ & $0.36 / 0.04$ & $93.1 / 1.7$ & $0.04 / 5.3$ \\
\hline $\mathrm{I}(4)$ & $-0.30 / 0.08$ & $0.04 / 0.08$ & $0.32 / 0.08$ & $88.0 / 7.8$ & $0.05 / 9.5$ \\
\hline \multicolumn{6}{|l|}{ 07/98 } \\
\hline $\mathrm{U}(5)$ & $-0.43 / 0.06$ & $-0.26 / 0.05$ & $0.51 / 0.06$ & $105.4 / 2.9$ & $0.08 / 3.6$ \\
\hline$B(5)$ & $-0.57 / 0.04$ & $-0.27 / 0.04$ & $0.64 / 0.04$ & $102.9 / 1.9$ & $0.07 / 4.2$ \\
\hline $\mathrm{V}(5)$ & $-0.60 / 0.06$ & $-0.19 / 0.08$ & $0.64 / 0.07$ & $98.8 / 3.0$ & $0.07 / 4.2$ \\
\hline$R(5)$ & $-0.59 / 0.05$ & $-0.19 / 0.07$ & $0.62 / 0.06$ & $98.9 / 2.8$ & $0.07 / 3.3$ \\
\hline$I(5)$ & $-0.56 / 0.07$ & $-0.18 / 0.06$ & $0.60 / 0.07$ & $99.2 / 3.1$ & $0.13 / 4.9$ \\
\hline
\end{tabular}


Table 13. Winter Be Star Summary

\begin{tabular}{|c|c|c|c|c|c|c|c|c|c|c|c|}
\hline $\begin{array}{c}\text { Star } \\
\text { Filter }\end{array}$ & $\begin{array}{l}q / d q \\
(\%)\end{array}$ & $\begin{array}{c}\langle d q> \\
(\%)\end{array}$ & $\begin{array}{l}u / d u \\
(\%)\end{array}$ & $\begin{array}{c}\langle d u> \\
(\%)\end{array}$ & $\begin{array}{c}p / d p \\
(\%)\end{array}$ & $\begin{array}{l}\langle d p> \\
(\%)\end{array}$ & $\begin{array}{c}\theta / d \theta \\
\left(^{\circ}\right)\end{array}$ & & $\begin{array}{c}\langle d \theta> \\
\left({ }^{\circ}\right)\end{array}$ & $\begin{array}{c}\langle d p i> \\
(\%)\end{array}$ & $\begin{array}{c}\langle d \theta i> \\
\left({ }^{\circ}\right)\end{array}$ \\
\hline \multicolumn{12}{|l|}{$\gamma$ Cas } \\
\hline $\mathrm{U}$ & $-0.47 / 0.08$ & 0.07 & $-0.28 / 0.03$ & 0.06 & $0.56 / 0.07$ & 0.08 & $105.7 /$ & 2.7 & 2.4 & 0.08 & 3.3 \\
\hline B & $-0.65 / 0.04$ & 0.05 & $-0.43 / 0.05$ & 0.08 & $0.79 / 0.06$ & 0.06 & $106.7 /$ & 1.0 & 2.7 & 0.06 & 2.5 \\
\hline $\mathrm{V}$ & $-0.58 / 0.01$ & 0.05 & $-0.37 / 0.04$ & 0.06 & $0.69 / 0.02$ & 0.05 & $106.3 /$ & 0.9 & 2.2 & 0.06 & 2.0 \\
\hline $\mathrm{R}$ & $-0.59 / 0.03$ & 0.07 & $-0.29 / 0.02$ & 0.07 & $0.66 / 0.03$ & 0.06 & $103.3 /$ & 0.6 & 3.5 & 0.08 & 2.6 \\
\hline I & $-0.47 / 0.05$ & 0.05 & $-0.23 / 0.02$ & 0.04 & $0.52 / 0.05$ & 0.05 & $103.2 /$ & 2.4 & 2.1 & 0.08 & 4.3 \\
\hline GAV & 0.04 & 0.06 & 0.03 & 0.06 & 0.05 & 0.06 & & 1.5 & 2.6 & 0.07 & 2.9 \\
\hline \multicolumn{12}{|l|}{$\phi$ Per } \\
\hline U & $0.16 / 0.09$ & 0.06 & $0.83 / 0.02$ & 0.10 & $0.86 / 0.02$ & 0.10 & $39.7 /$ & 2.9 & 2.1 & 0.08 & 2.9 \\
\hline B & $0.47 / 0.05$ & 0.06 & $1.24 / 0.11$ & 0.08 & $1.33 / 0.11$ & 0.07 & $34.5 /$ & 0.9 & 1.4 & 0.05 & 1.4 \\
\hline V & $0.38 / 0.04$ & 0.06 & $1.11 / 0.09$ & 0.07 & $1.17 / 0.10$ & 0.08 & $35.4 /$ & 0.3 & 1.3 & 0.06 & 1.6 \\
\hline $\mathrm{R}$ & $0.27 / 0.04$ & 0.04 & $0.99 / 0.08$ & 0.09 & $1.02 / 0.09$ & 0.08 & $37.3 /$ & 0.5 & 1.4 & 0.05 & 2.1 \\
\hline I & $0.20 / 0.08$ & 0.06 & $0.79 / 0.12$ & 0.09 & $0.82 / 0.13$ & 0.09 & $38.0 /$ & 2.1 & 1.8 & 0.09 & 2.8 \\
\hline GAV & 0.06 & 0.06 & 0.08 & 0.09 & 0.09 & 0.09 & & 1.4 & 1.6 & 0.07 & 2.2 \\
\hline \multicolumn{12}{|l|}{48 Per } \\
\hline $\mathrm{U}$ & $0.73 / 0.09$ & 0.06 & $-0.20 / 0.05$ & 0.06 & $0.76 / 0.10$ & 0.06 & $172.0 /$ & 1.0 & 2.5 & 0.09 & 2.6 \\
\hline B & $0.83 / 0.06$ & 0.04 & $-0.24 / 0.04$ & 0.05 & $0.87 / 0.05$ & 0.03 & $171.9 /$ & 1.5 & 1.7 & 0.05 & 1.8 \\
\hline V & $0.93 / 0.04$ & 0.07 & $-0.25 / 0.06$ & 0.05 & $0.97 / 0.04$ & 0.06 & $172.4 /$ & 1.7 & 1.8 & 0.07 & 2.3 \\
\hline $\mathrm{R}$ & $0.91 / 0.05$ & 0.04 & $-0.24 / 0.04$ & 0.05 & $0.95 / 0.04$ & 0.04 & $172.6 /$ & 1.5 & 1.8 & 0.07 & 1.6 \\
\hline I & $0.82 / 0.07$ & 0.08 & $-0.20 / 0.04$ & 0.06 & $0.85 / 0.07$ & 0.07 & $172.8 /$ & 1.7 & 2.4 & 0.09 & 2.8 \\
\hline GAV & 0.06 & 0.06 & 0.04 & 0.06 & 0.06 & 0.05 & & 1.5 & 2.0 & 0.07 & 2.2 \\
\hline \multicolumn{12}{|l|}{$\zeta$ Tau } \\
\hline U & $0.51 / 0.06$ & 0.05 & $1.06 / 0.05$ & 0.06 & $1.19 / 0.06$ & 0.05 & $32.2 /$ & 1.4 & 1.4 & 0.07 & 2.0 \\
\hline B & $0.65 / 0.07$ & 0.05 & $1.36 / 0.09$ & 0.09 & $1.51 / 0.10$ & 0.08 & $32.2 /$ & 1.0 & 1.0 & 0.05 & 1.8 \\
\hline $\mathrm{V}$ & $0.63 / 0.06$ & 0.05 & $1.29 / 0.06$ & 0.07 & $1.44 / 0.08$ & 0.06 & $32.0 /$ & 0.7 & 1.1 & 0.06 & 1.6 \\
\hline $\mathrm{R}$ & $0.52 / 0.03$ & 0.04 & $1.23 / 0.07$ & 0.05 & $1.34 / 0.07$ & 0.05 & $33.5 /$ & 0.2 & 0.9 & 0.08 & 1.6 \\
\hline I & $0.46 / 0.11$ & 0.04 & $1.06 / 0.05$ & 0.11 & $1.16 / 0.07$ & 0.11 & $33.3 /$ & 2.4 & 1.0 & 0.08 & 2.0 \\
\hline GAV & 0.07 & 0.04 & 0.06 & 0.08 & 0.08 & 0.07 & & 1.1 & 1.1 & 0.07 & 1.8 \\
\hline
\end{tabular}


Table 14. Summer Be Star Summary

\begin{tabular}{|c|c|c|c|c|c|c|c|c|c|c|}
\hline $\begin{array}{c}\text { Star } \\
\text { Filter }\end{array}$ & $\begin{array}{c}q / d q \\
(\%)\end{array}$ & $\begin{array}{c}\langle d q> \\
(\%)\end{array}$ & $\begin{array}{c}u / d u \\
(\%)\end{array}$ & $\begin{array}{c}\langle d u> \\
(\%)\end{array}$ & $\begin{array}{c}p / d p \\
(\%)\end{array}$ & $\begin{array}{c}<d p> \\
(\%)\end{array}$ & $\begin{array}{c}\theta / d \theta \\
\left(^{\circ}\right)\end{array}$ & $\begin{array}{c}\langle d \theta> \\
\left({ }^{\circ}\right)\end{array}$ & $\begin{array}{c}<d p i> \\
(\%)\end{array}$ & $\begin{array}{c}\langle d \theta i> \\
\left({ }^{\circ}\right)\end{array}$ \\
\hline \multicolumn{11}{|l|}{$48 \mathrm{Lib}$} \\
\hline $\mathrm{U}$ & $-0.38 / 0.04$ & 0.08 & $-0.56 / 0.12$ & 0.08 & $0.69 / 0.11$ & 0.08 & $117.6 / 1.9$ & 3.6 & 0.12 & 5.1 \\
\hline B & $-0.41 / 0.04$ & 0.05 & $-0.80 / 0.06$ & 0.07 & $0.90 / 0.04$ & 0.06 & $121.3 / 2.2$ & 1.8 & 0.06 & 2.8 \\
\hline $\mathrm{V}$ & $-0.52 / 0.06$ & 0.04 & $-0.70 / 0.03$ & 0.06 & $0.88 / 0.02$ & 0.07 & $116.7 / 2.1$ & 1.2 & 0.07 & 2.2 \\
\hline $\mathrm{R}$ & $-0.52 / 0.06$ & 0.06 & $-0.62 / 0.02$ & 0.05 & $0.82 / 0.05$ & 0.05 & $115.1 / 1.3$ & 1.9 & 0.08 & 2.4 \\
\hline I & $-0.53 / 0.10$ & 0.05 & $-0.56 / 0.04$ & 0.10 & $0.79 / 0.08$ & 0.09 & $113.3 / 2.6$ & 3.1 & 0.12 & 5.6 \\
\hline GAV & 0.06 & 0.06 & 0.06 & 0.07 & 0.06 & 0.07 & 2.0 & 2.3 & 0.09 & 3.6 \\
\hline \multicolumn{11}{|l|}{$\chi 0 \mathrm{ph}$} \\
\hline $\mathrm{U}$ & $0.04 / 0.06$ & 0.12 & $-0.33 / 0.06$ & 0.05 & $0.39 / 0.02$ & 0.06 & $138.0 / 4.7$ & 10.2 & 0.11 & 13.4 \\
\hline B & $-0.02 / 0.03$ & 0.08 & $-0.46 / 0.04$ & 0.06 & $0.48 / 0.04$ & 0.06 & $134.6 / 1.7$ & 5.8 & 0.09 & 5.9 \\
\hline $\mathrm{V}$ & $-0.00 / 0.02$ & 0.09 & $-0.47 / 0.04$ & 0.06 & $0.49 / 0.03$ & 0.06 & $135.0 / 1.2$ & 5.5 & 0.10 & 5.7 \\
\hline $\mathrm{R}$ & $-0.01 / 0.05$ & 0.08 & $-0.47 / 0.01$ & 0.06 & $0.48 / 0.01$ & 0.05 & $134.7 / 3.1$ & 4.8 & 0.08 & 6.4 \\
\hline $\mathrm{I}$ & $-0.05 / 0.07$ & 0.09 & $-0.42 / 0.03$ & 0.08 & $0.46 / 0.04$ & 0.06 & $133.0 / 4.7$ & 7.0 & 0.12 & 9.8 \\
\hline GAV & 0.05 & 0.09 & 0.04 & 0.06 & 0.03 & 0.06 & 3.1 & 6.7 & 0.10 & 8.2 \\
\hline \multicolumn{11}{|l|}{$\pi$ Aqr } \\
\hline $\mathrm{U}$ & $-0.25 / 0.16$ & 0.06 & $-0.33 / 0.08$ & 0.09 & $0.46 / 0.04$ & 0.09 & $116.1 / 10.6$ & 4.2 & 0.11 & 7.9 \\
\hline B & $-0.30 / 0.14$ & 0.05 & $-0.37 / 0.09$ & 0.04 & $0.50 / 0.01$ & 0.04 & $115.6 / 9.7$ & 2.8 & 0.08 & 4.0 \\
\hline V & $-0.32 / 0.17$ & 0.06 & $-0.35 / 0.09$ & 0.04 & $0.51 / 0.01$ & 0.03 & $114.3 / 11.1$ & 3.7 & 0.07 & 3.9 \\
\hline $\mathrm{R}$ & $-0.30 / 0.17$ & 0.06 & $-0.33 / 0.08$ & 0.04 & $0.48 / 0.02$ & 0.05 & $114.6 / 11.7$ & 3.0 & 0.08 & 4.1 \\
\hline I & $-0.22 / 0.18$ & 0.09 & $-0.31 / 0.07$ & 0.05 & $0.44 / 0.02$ & 0.07 & $118.3 / 13.1$ & 6.1 & 0.09 & 9.7 \\
\hline GAV & 0.16 & 0.06 & 0.08 & 0.05 & 0.02 & 0.05 & 11.2 & 4.0 & 0.09 & 5.9 \\
\hline \multicolumn{11}{|l|}{ o And } \\
\hline $\mathrm{U}$ & $-0.39 / 0.09$ & 0.05 & $-0.11 / 0.11$ & 0.05 & $0.42 / 0.11$ & 0.04 & $96.3 / 6.6$ & 3.9 & 0.08 & 5.7 \\
\hline B & $-0.46 / 0.12$ & 0.04 & $-0.13 / 0.11$ & 0.06 & $0.49 / 0.14$ & 0.05 & $97.1 / 4.3$ & 3.6 & 0.06 & 3.9 \\
\hline $\mathrm{V}$ & $-0.45 / 0.13$ & 0.05 & $-0.08 / 0.07$ & 0.04 & $0.47 / 0.14$ & 0.05 & $94.8 / 3.6$ & 2.6 & 0.06 & 5.4 \\
\hline $\mathrm{R}$ & $-0.43 / 0.13$ & 0.05 & $-0.08 / 0.07$ & 0.04 & $0.45 / 0.14$ & 0.05 & $94.8 / 3.1$ & 2.4 & 0.05 & 5.3 \\
\hline I & $-0.40 / 0.13$ & 0.08 & $-0.06 / 0.09$ & 0.07 & $0.43 / 0.14$ & 0.09 & $93.8 / 4.6$ & 5.1 & 0.08 & 7.7 \\
\hline GAV & 0.12 & 0.05 & 0.09 & 0.05 & 0.13 & 0.05 & 4.5 & 3.5 & 0.07 & 5.6 \\
\hline
\end{tabular}


Table 15. Polarized Standard Star Summary (Limber)

\begin{tabular}{|c|c|c|c|c|c|c|c|c|c|c|c|}
\hline $\begin{array}{l}\text { Star } \\
\text { Filter }\end{array}$ & $\begin{array}{l}q / d q \\
(\%)\end{array}$ & $\begin{array}{c}\langle d q> \\
(\%)\end{array}$ & $\begin{array}{c}u / d u \\
(\%)\end{array}$ & $\begin{array}{c}\langle d u> \\
(\%)\end{array}$ & $\begin{array}{c}p / d p \\
(\%)\end{array}$ & $\begin{array}{c}\langle d p> \\
(\%)\end{array}$ & $\begin{array}{c}\theta / d \theta \\
\left({ }^{\circ}\right)\end{array}$ & & $\begin{array}{c}\langle d \theta\rangle \\
\left({ }^{\circ}\right)\end{array}$ & $\begin{array}{c}\langle d p i> \\
(\%)\end{array}$ & $\begin{array}{c}\langle d \theta i> \\
\left({ }^{\circ}\right)\end{array}$ \\
\hline \multicolumn{12}{|l|}{ 2H Cam } \\
\hline $\mathrm{U}$ & $-1.83 / 0.09$ & 0.04 & $-2.41 / 0.06$ & 0.04 & $3.03 / 0.07$ & 0.03 & $116.5 /$ & 0.8 & 0.5 & 0.09 & 1.1 \\
\hline B & $-1.95 / 0.08$ & 0.07 & $-2.65 / 0.06$ & 0.05 & $3.29 / 0.02$ & 0.06 & $116.8 /$ & 0.9 & 0.6 & 0.06 & 0.6 \\
\hline $\mathrm{V}$ & $-2.05 / 0.02$ & 0.06 & $-2.75 / 0.04$ & 0.06 & $3.43 / 0.02$ & 0.06 & $116.7 /$ & 0.4 & 0.6 & 0.06 & 0.6 \\
\hline $\mathrm{R}$ & $-1.98 / 0.02$ & 0.05 & $-2.66 / 0.04$ & 0.06 & $3.32 / 0.02$ & 0.05 & $116.7 /$ & 0.3 & 0.6 & 0.05 & 0.5 \\
\hline I & $-1.79 / 0.03$ & 0.07 & $-2.37 / 0.09$ & 0.06 & $2.96 / 0.06$ & 0.08 & $116.4 /$ & 0.6 & 0.7 & 0.09 & 0.8 \\
\hline GAV & 0.05 & 0.06 & 0.06 & 0.06 & 0.04 & 0.06 & & 0.6 & 0.6 & 0.07 & 0.7 \\
\hline \multicolumn{12}{|l|}{ o Sco } \\
\hline $\mathrm{U}$ & $1.06 / 0.14$ & 0.18 & $2.35 / 0.05$ & 0.23 & $2.59 / 0.10$ & 0.25 & $33.0 /$ & 1.3 & 1.7 & 0.19 & 2.7 \\
\hline B & $1.41 / 0.11$ & 0.07 & $3.09 / 0.06$ & 0.06 & $3.40 / 0.04$ & 0.08 & $32.8 /$ & 1.0 & 0.5 & 0.08 & 0.8 \\
\hline $\mathrm{V}$ & $1.73 / 0.16$ & 0.08 & $3.76 / 0.05$ & 0.08 & $4.15 / 0.05$ & 0.08 & $32.7 /$ & 1.1 & 0.5 & 0.10 & 0.8 \\
\hline $\mathrm{R}$ & $1.83 / 0.16$ & 0.07 & $4.00 / 0.06$ & 0.07 & $4.40 / 0.04$ & 0.08 & $32.8 /$ & 1.1 & 0.4 & 0.09 & 0.6 \\
\hline I & $1.74 / 0.15$ & 0.07 & $3.93 / 0.10$ & 0.05 & $4.30 / 0.05$ & 0.05 & $33.1 /$ & 1.2 & 0.5 & 0.14 & 1.0 \\
\hline GAV & 0.14 & 0.10 & 0.06 & 0.10 & 0.06 & 0.11 & & 1.1 & 0.7 & 0.12 & 1.2 \\
\hline
\end{tabular}

Table 16. Polarized Standard Star Summary (McDonald)

\begin{tabular}{|c|c|c|c|c|c|c|c|c|c|c|}
\hline $\begin{array}{l}\text { Star } \\
\text { Filter }\end{array}$ & $\begin{array}{c}q / d q \\
(\%)\end{array}$ & $\begin{array}{c}<d q> \\
(\%)\end{array}$ & $\begin{array}{c}u / d u \\
(\%)\end{array}$ & $\begin{array}{c}\langle d u> \\
(\%)\end{array}$ & $\begin{array}{c}p / d p \\
(\%)\end{array}$ & $\begin{array}{c}\langle d p> \\
(\%)\end{array}$ & $\begin{array}{c}\theta / d \theta \\
\left(^{\circ}\right)\end{array}$ & $\begin{array}{c}\langle d \theta> \\
\left({ }^{\circ}\right)\end{array}$ & $\begin{array}{c}\langle d p i> \\
(\%)\end{array}$ & $\begin{array}{c}\langle d \theta i> \\
\left({ }^{\circ}\right)\end{array}$ \\
\hline \multicolumn{11}{|l|}{ 2H Cam } \\
\hline $\mathrm{U}$ & $-1.83 / 0.05$ & 0.11 & $-2.41 / 0.03$ & 0.07 & $3.03 / 0.01$ & 0.08 & $116.5 / 0.6$ & 1.1 & 0.09 & 0.8 \\
\hline B & $-1.97 / 0.05$ & 0.07 & $-2.60 / 0.09$ & 0.07 & $3.26 / 0.07$ & 0.05 & $116.3 / 0.6$ & 0.7 & 0.04 & 0.4 \\
\hline $\mathrm{V}$ & $-2.07 / 0.06$ & 0.07 & $-2.73 / 0.01$ & 0.07 & $3.43 / 0.03$ & 0.07 & $116.4 / 0.5$ & 0.5 & 0.04 & 0.4 \\
\hline $\mathrm{R}$ & $-1.97 / 0.07$ & 0.05 & $-2.66 / 0.05$ & 0.06 & $3.31 / 0.04$ & 0.05 & $116.8 / 0.7$ & 0.4 & 0.03 & 0.3 \\
\hline I & $-1.75 / 0.06$ & 0.09 & $-2.36 / 0.05$ & 0.06 & $2.94 / 0.05$ & 0.08 & $116.8 / 0.7$ & 0.8 & 0.05 & 0.6 \\
\hline GAV & 0.06 & 0.08 & 0.05 & 0.07 & 0.04 & 0.07 & 0.6 & 0.7 & 0.05 & 0.5 \\
\hline \multicolumn{11}{|l|}{ o Sco } \\
\hline $\mathrm{U}$ & $1.35 / 0.21$ & 0.20 & $2.51 / 0.12$ & 0.25 & $2.85 / 0.12$ & 0.28 & $30.9 / 2.0$ & 1.6 & 0.20 & 2.1 \\
\hline B & $1.40 / 0.12$ & 0.09 & $3.07 / 0.14$ & 0.08 & $3.39 / 0.10$ & 0.07 & $32.7 / 1.3$ & 0.9 & 0.06 & 0.5 \\
\hline $\mathrm{V}$ & $1.81 / 0.25$ & 0.12 & $3.74 / 0.12$ & 0.08 & $4.16 / 0.01$ & 0.10 & $32.1 / 1.9$ & 0.7 & 0.05 & 0.3 \\
\hline $\mathrm{R}$ & $1.91 / 0.23$ & 0.07 & $3.96 / 0.10$ & 0.08 & $4.41 / 0.03$ & 0.05 & $32.1 / 1.6$ & 0.6 & 0.04 & 0.2 \\
\hline I & $1.76 / 0.19$ & 0.07 & $3.88 / 0.06$ & 0.06 & $4.26 / 0.06$ & 0.07 & $32.8 / 1.3$ & 0.4 & 0.05 & 0.3 \\
\hline GAV & 0.20 & 0.11 & 0.11 & 0.11 & 0.06 & 0.11 & 1.6 & 0.9 & 0.08 & 0.7 \\
\hline
\end{tabular}


Table 17. Interstellar Polarization Parameters

\begin{tabular}{lccrl}
\hline \hline Star & $\begin{array}{c}p_{\max } \\
(\%)\end{array}$ & $\begin{array}{c}\lambda_{\max } \\
(\AA)\end{array}$ & $\begin{array}{r}\theta_{I S} \\
\left(^{\circ}\right)\end{array}$ & Reference \\
\hline$\gamma$ Cas & 0.26 & 5900 & 95 & MB \\
$\phi$ Per & 1.06 & 4460 & 106 & PBL \\
48 Per & 0.85 & 5990 & 0 & PBL \\
$\zeta$ Tau & 0.00 & $\ldots$ & $\ldots$ & PBL \\
48 Lib & 0.64 & 5670 & 85 & PBL \\
$\chi$ Oph & 0.37 & 5740 & 158 & PBL \\
$\pi$ Aqr & 0.46 & 4980 & 116 & PBL \\
$\circ$ And & 0.26 & 5970 & 75 & PBL \\
\hline
\end{tabular}



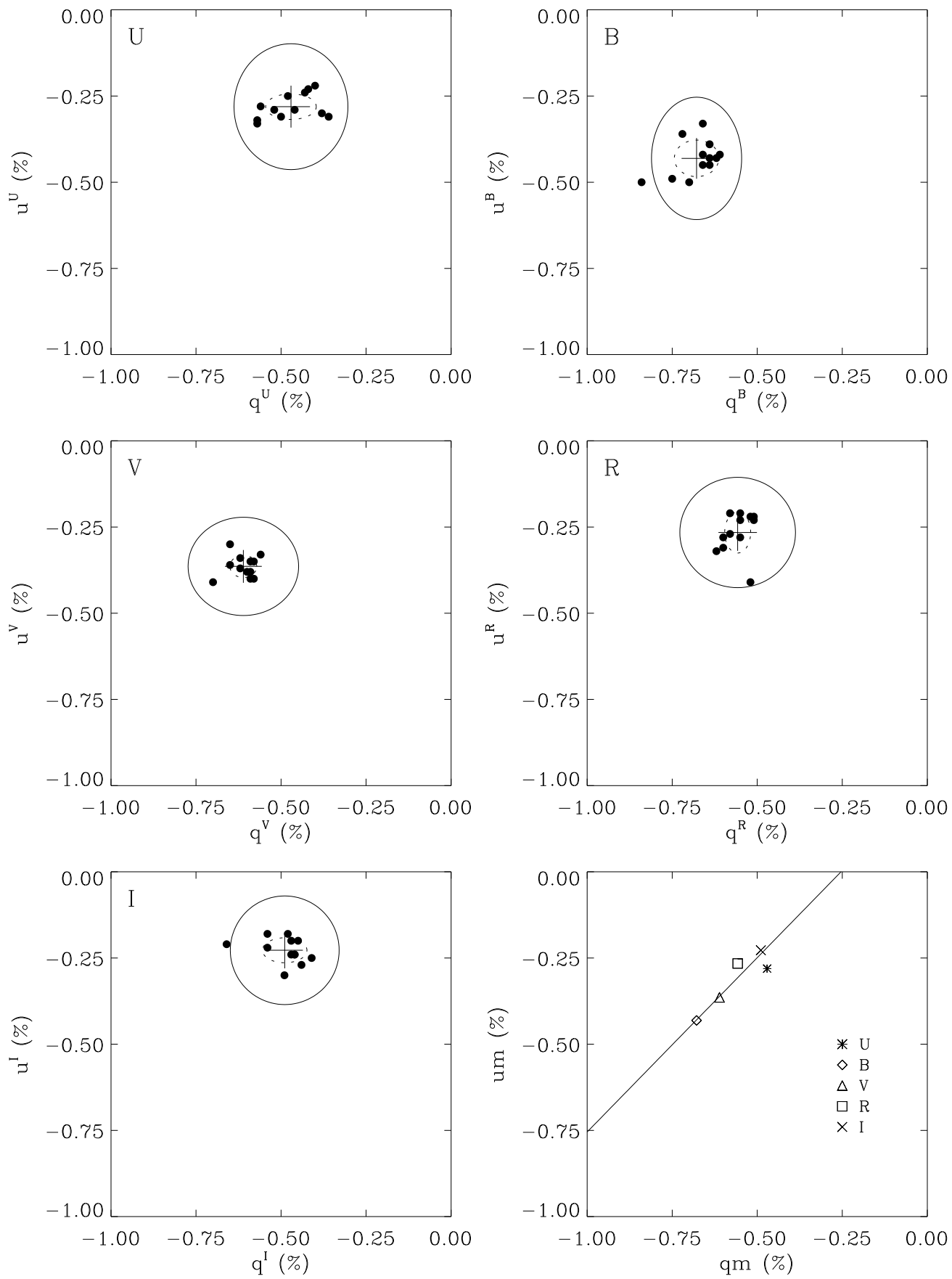


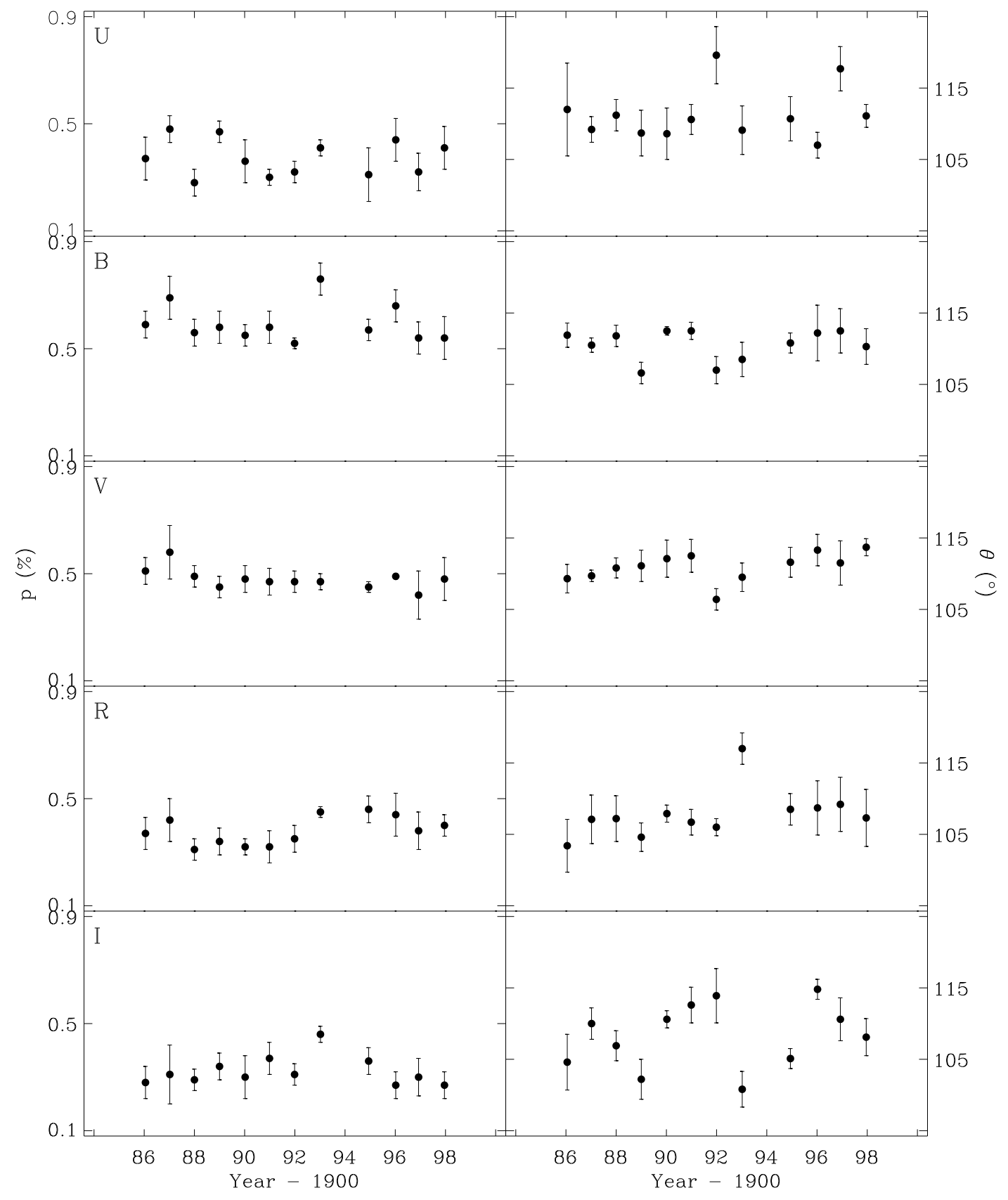



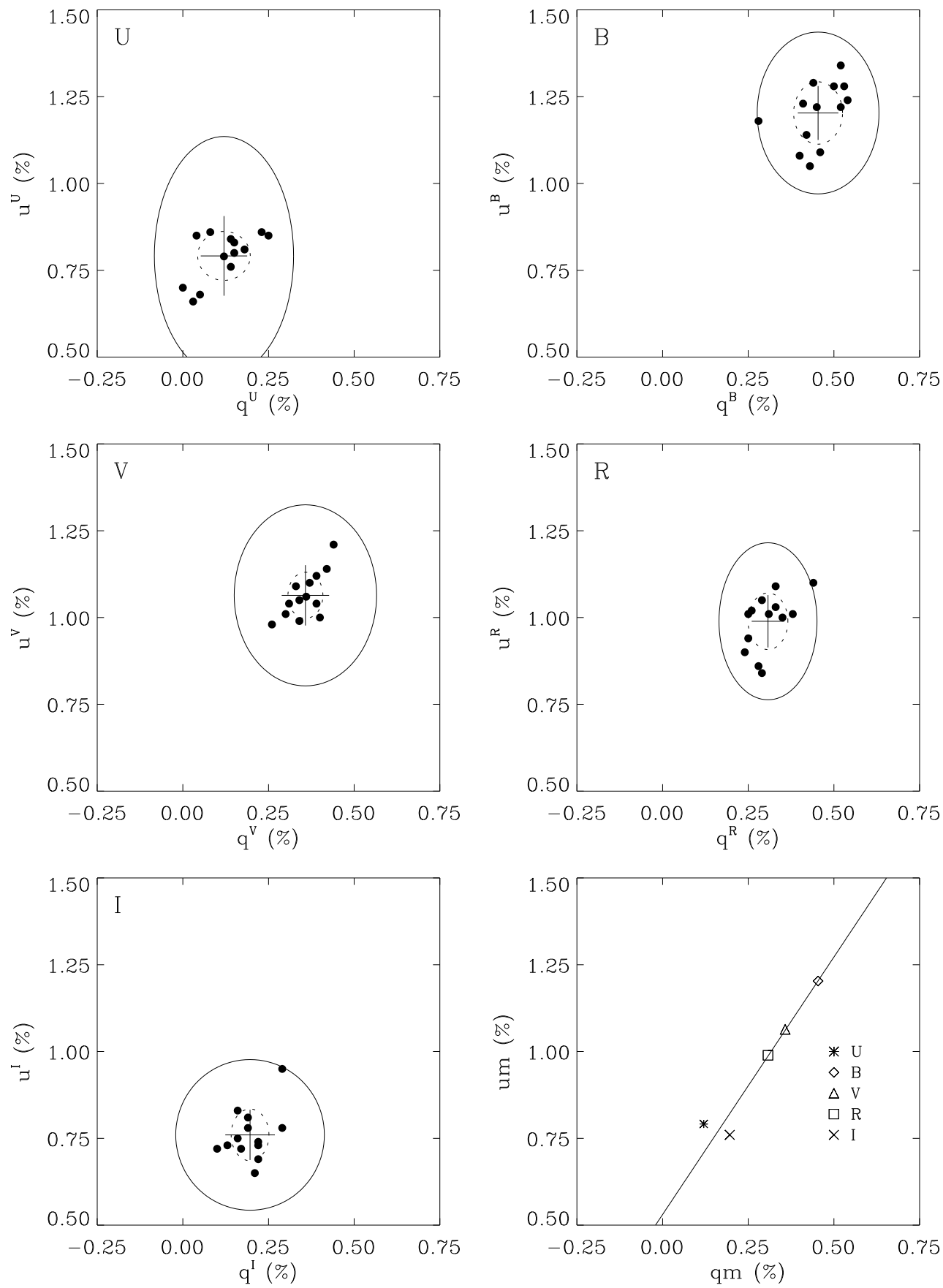


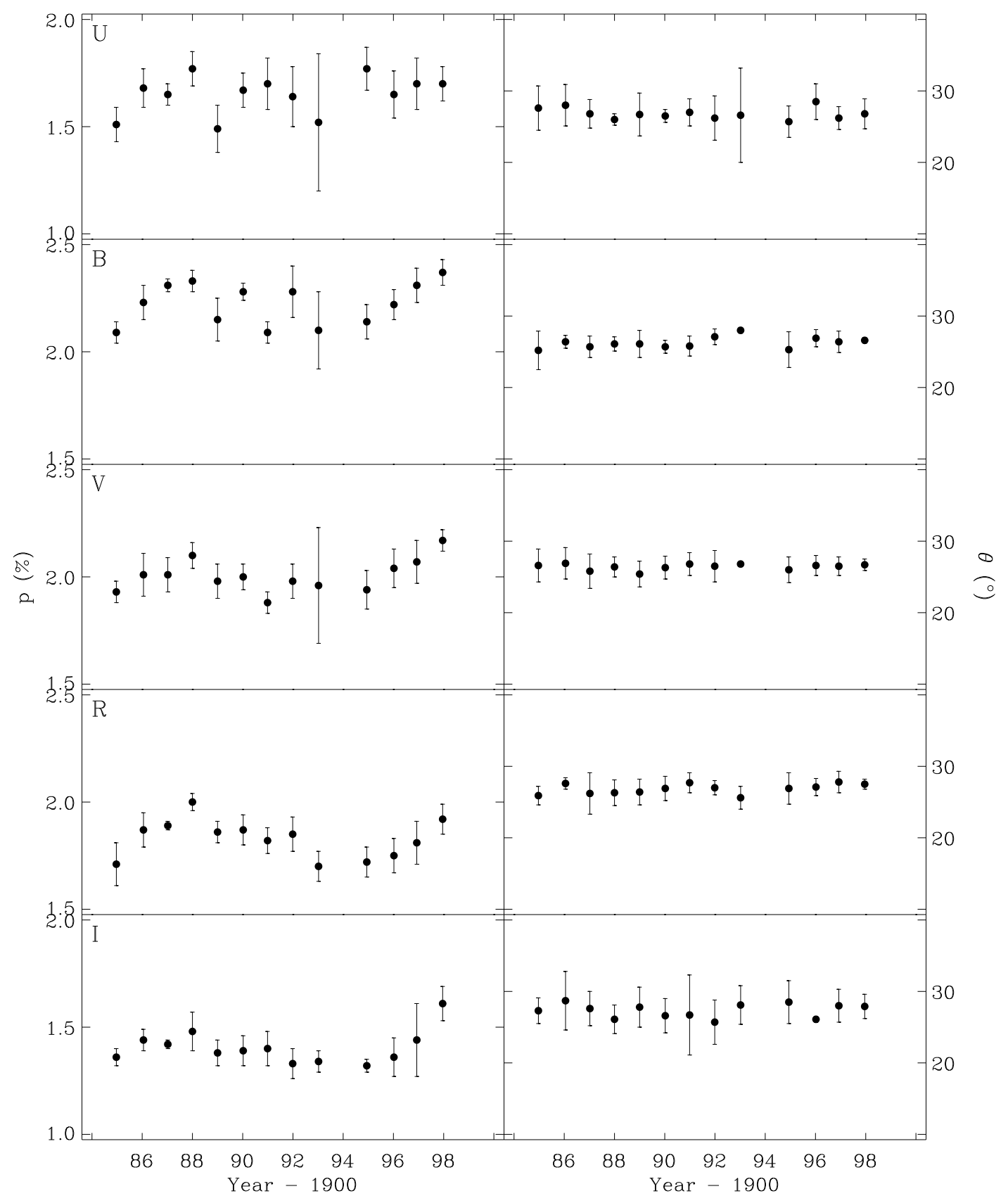



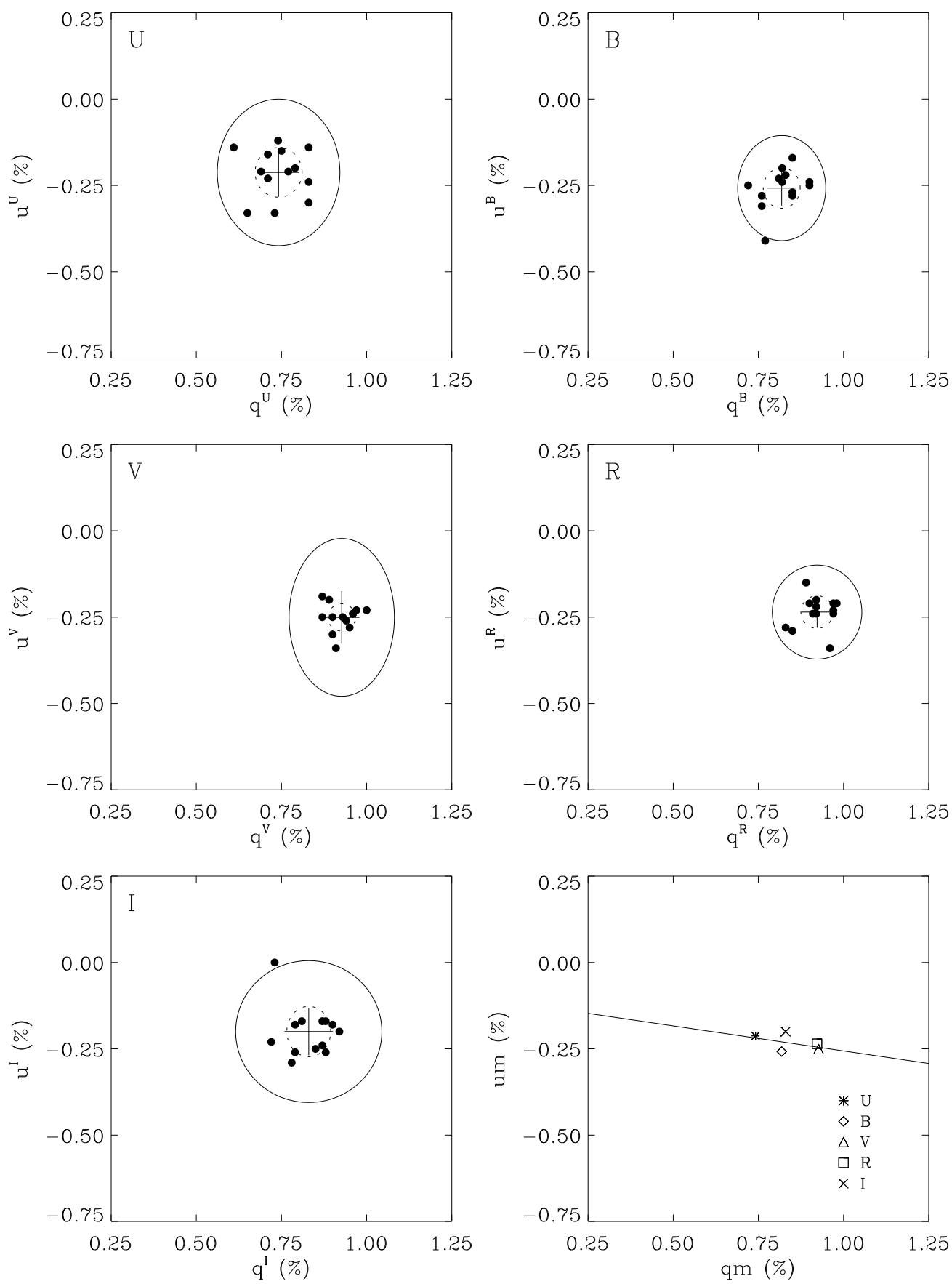


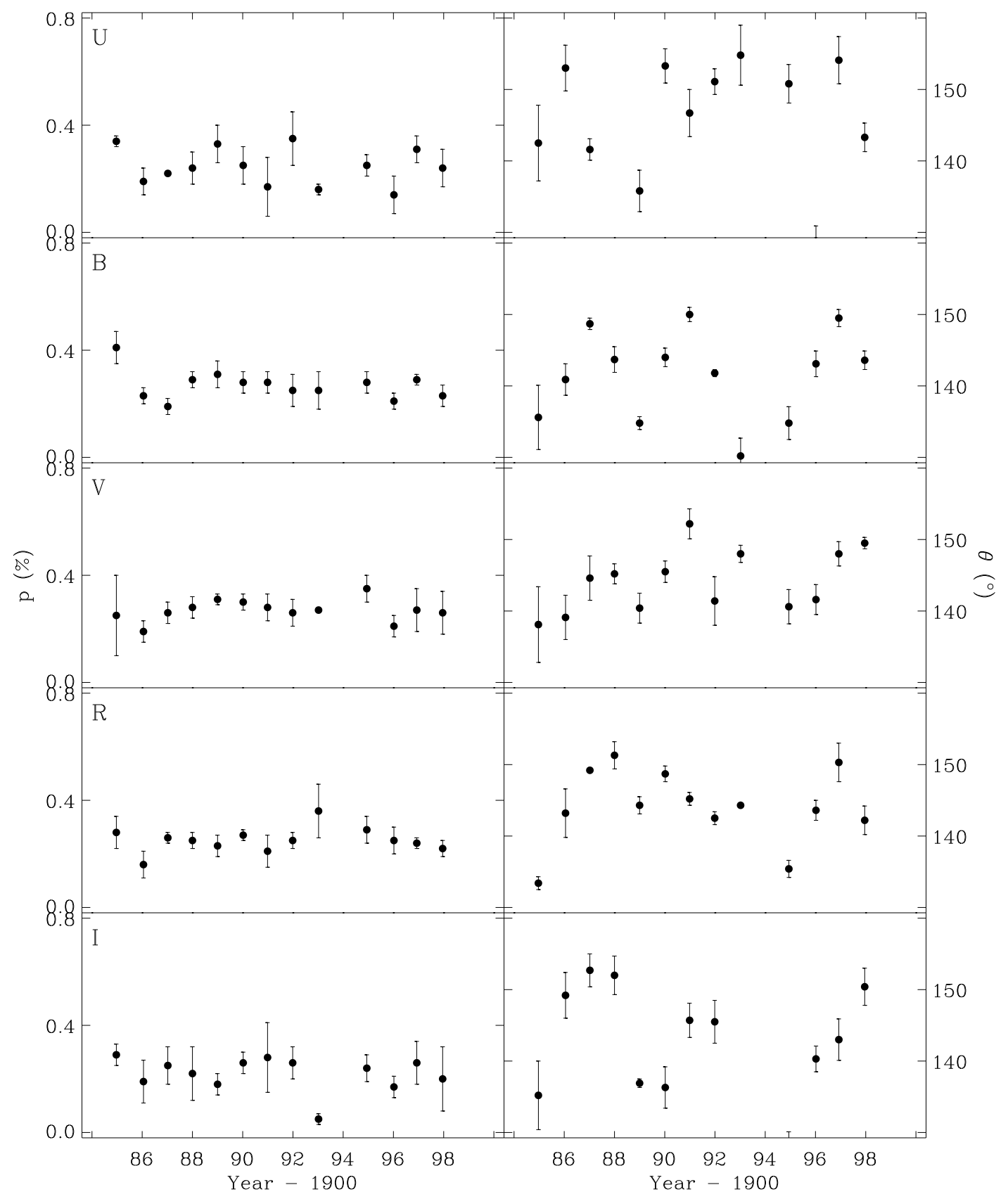



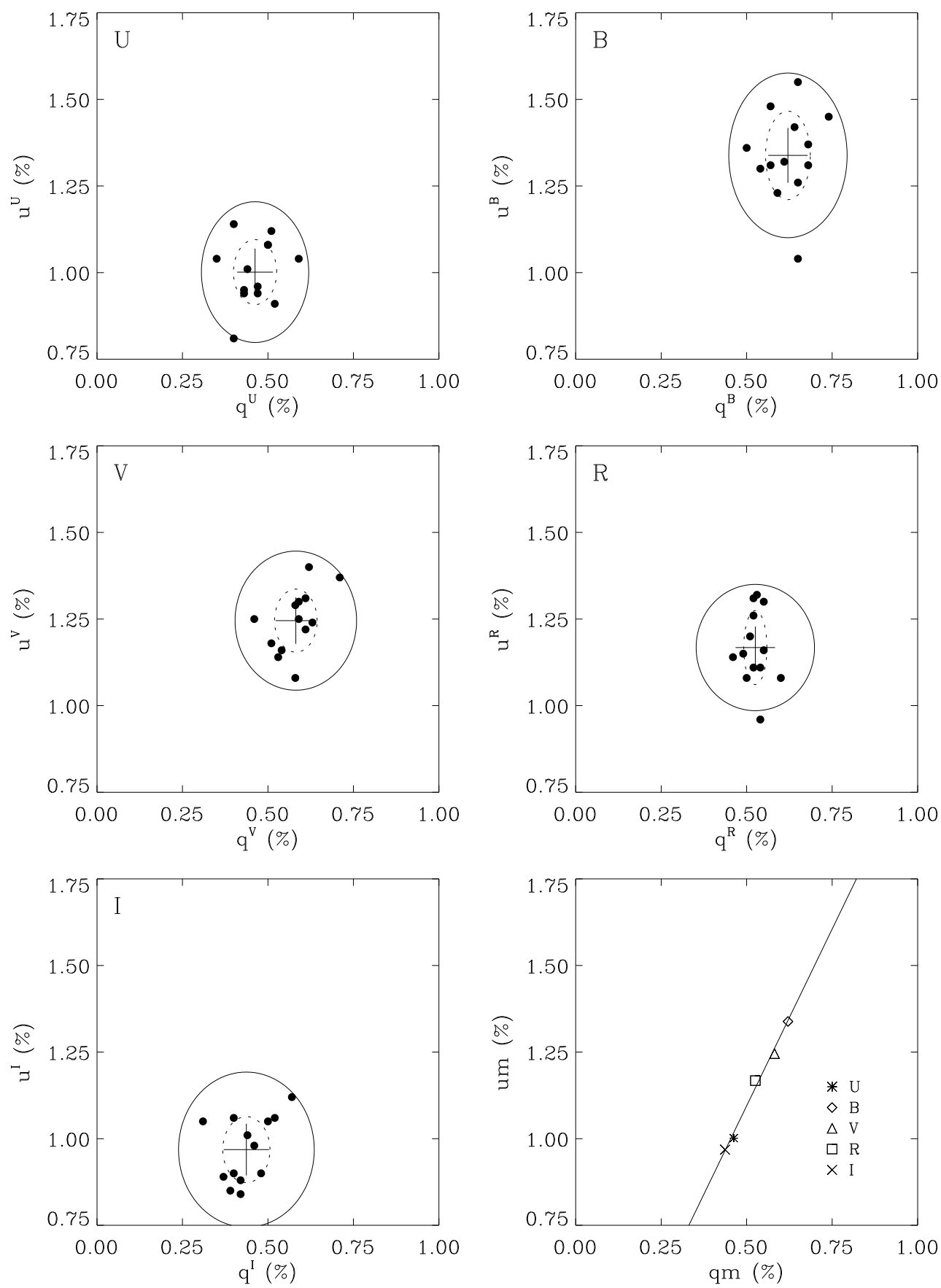


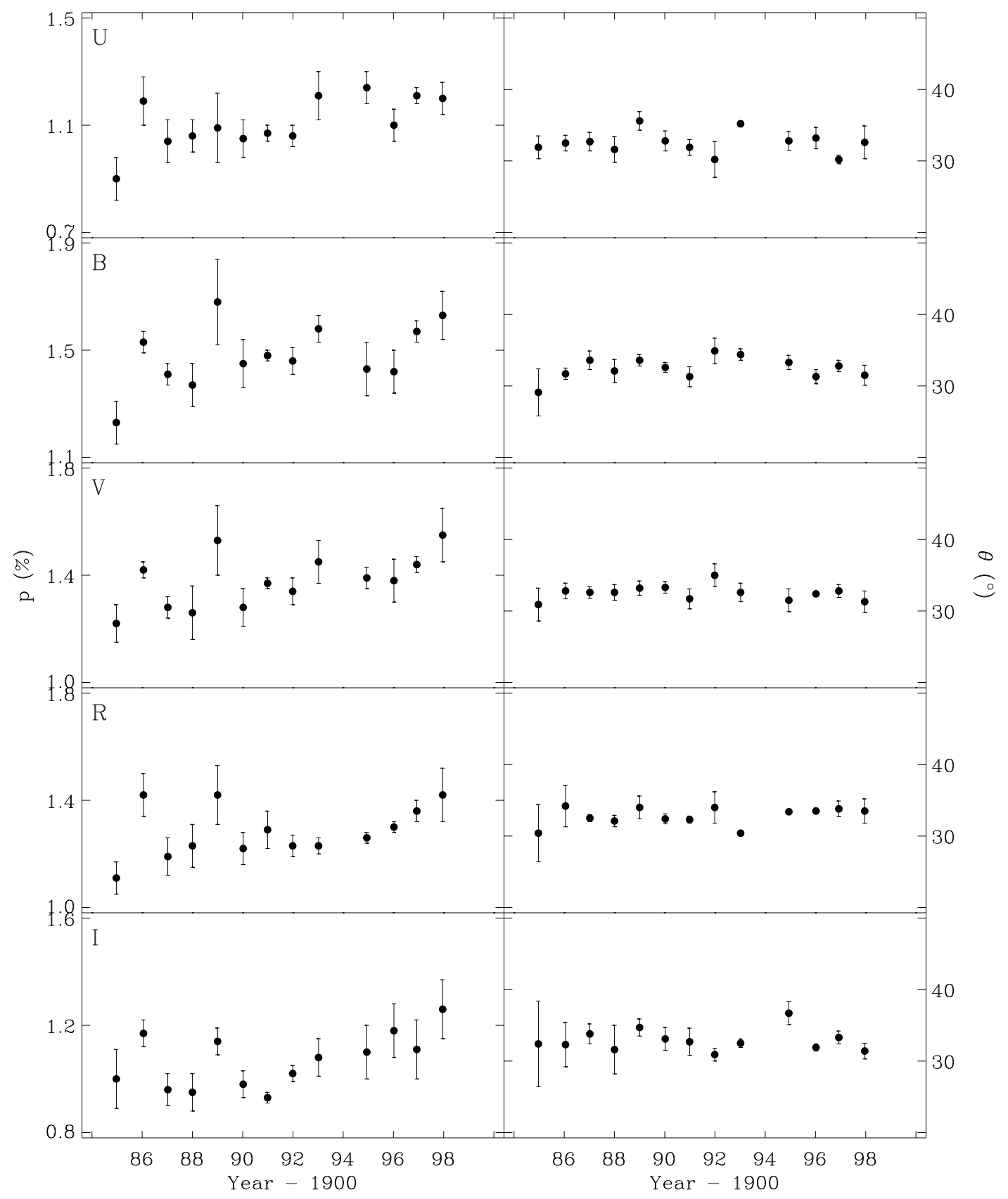



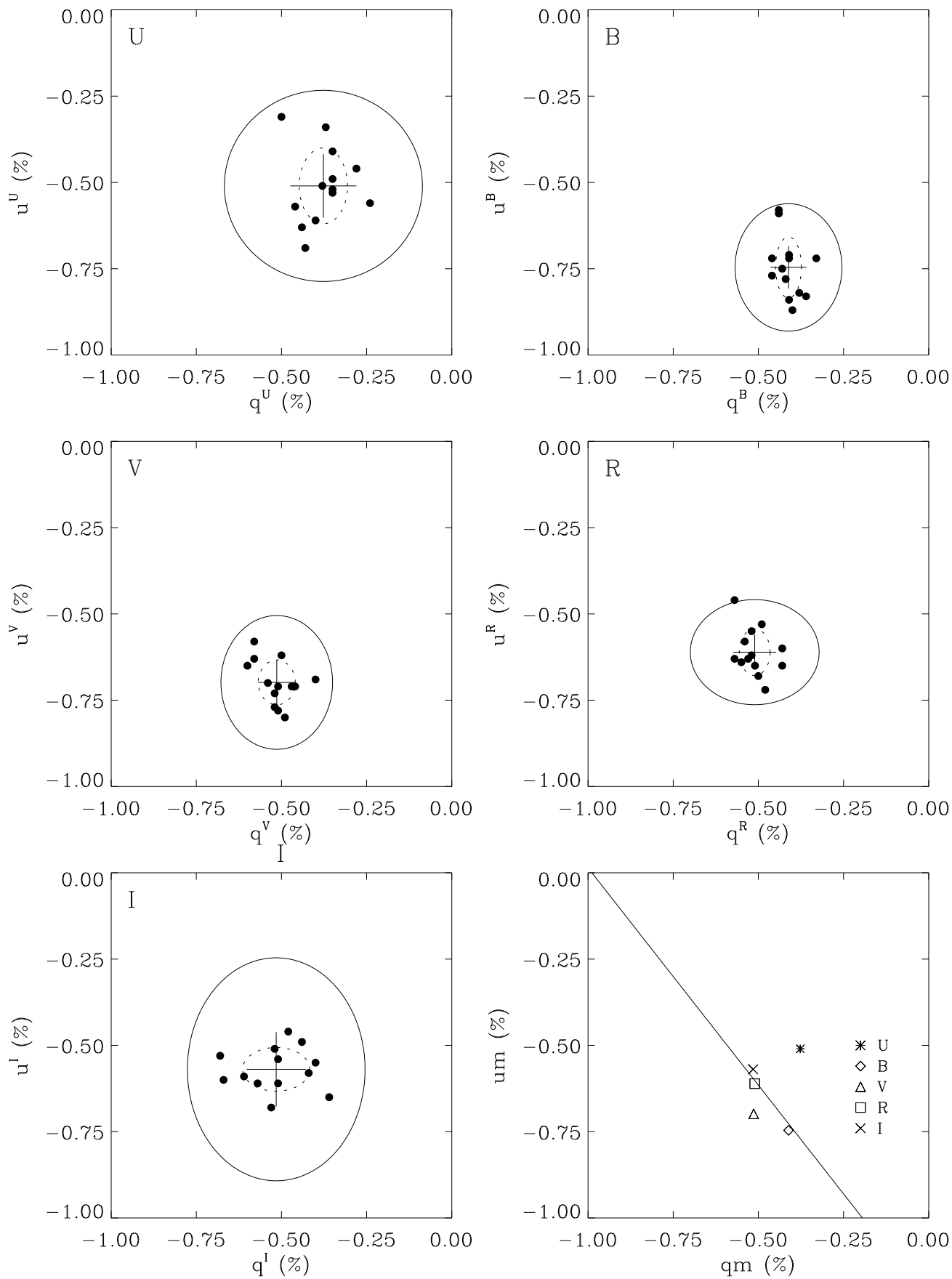

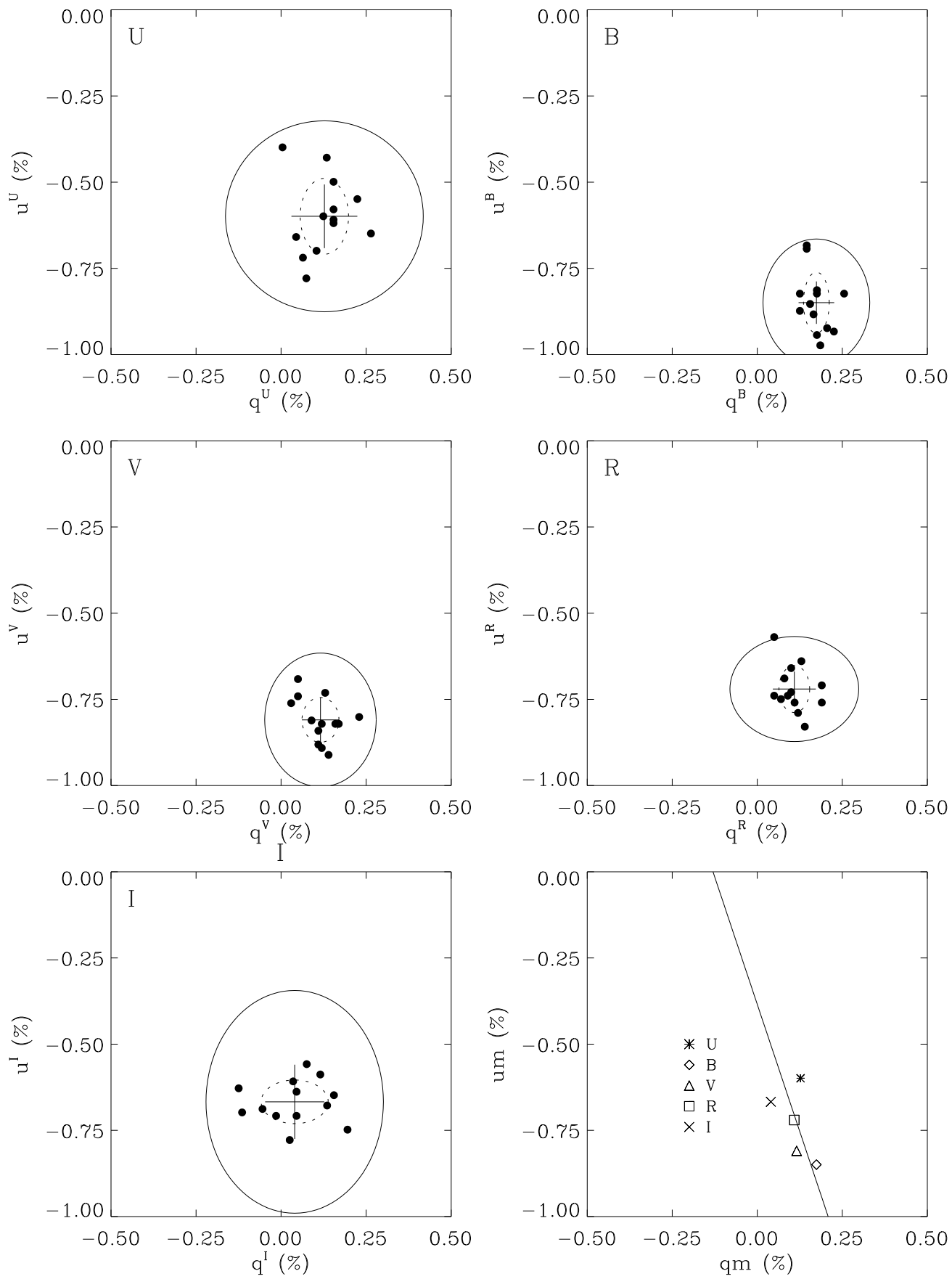


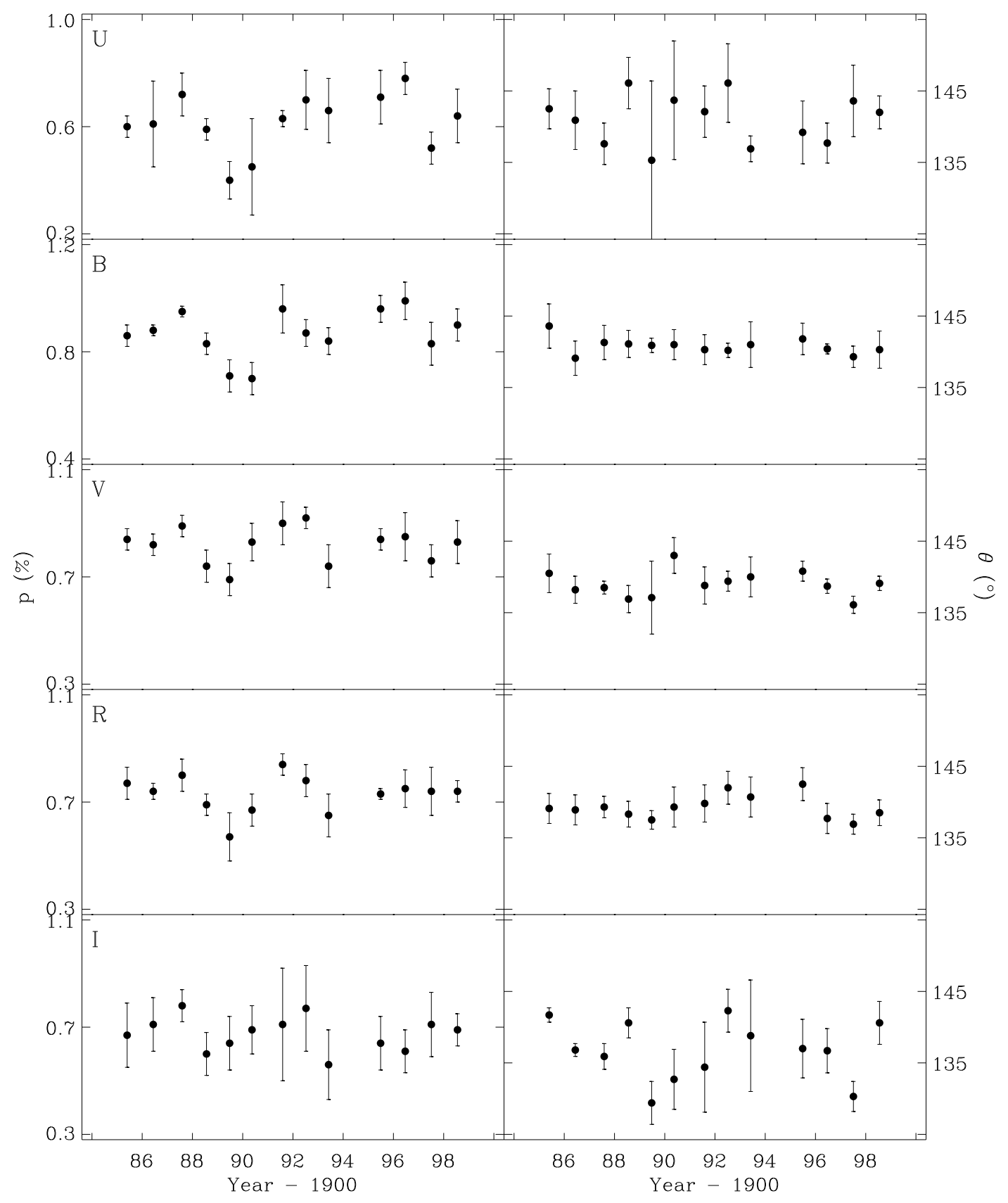



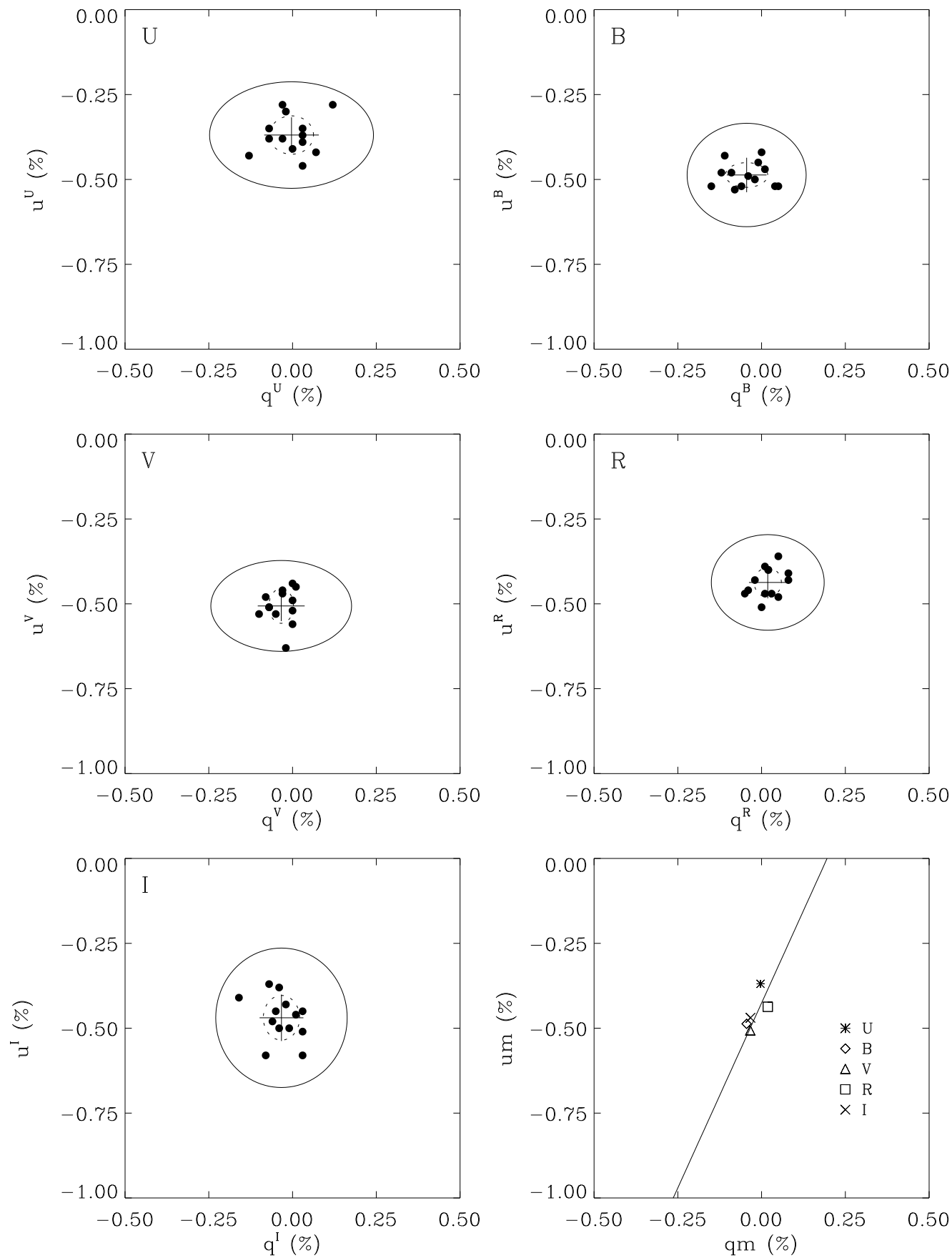


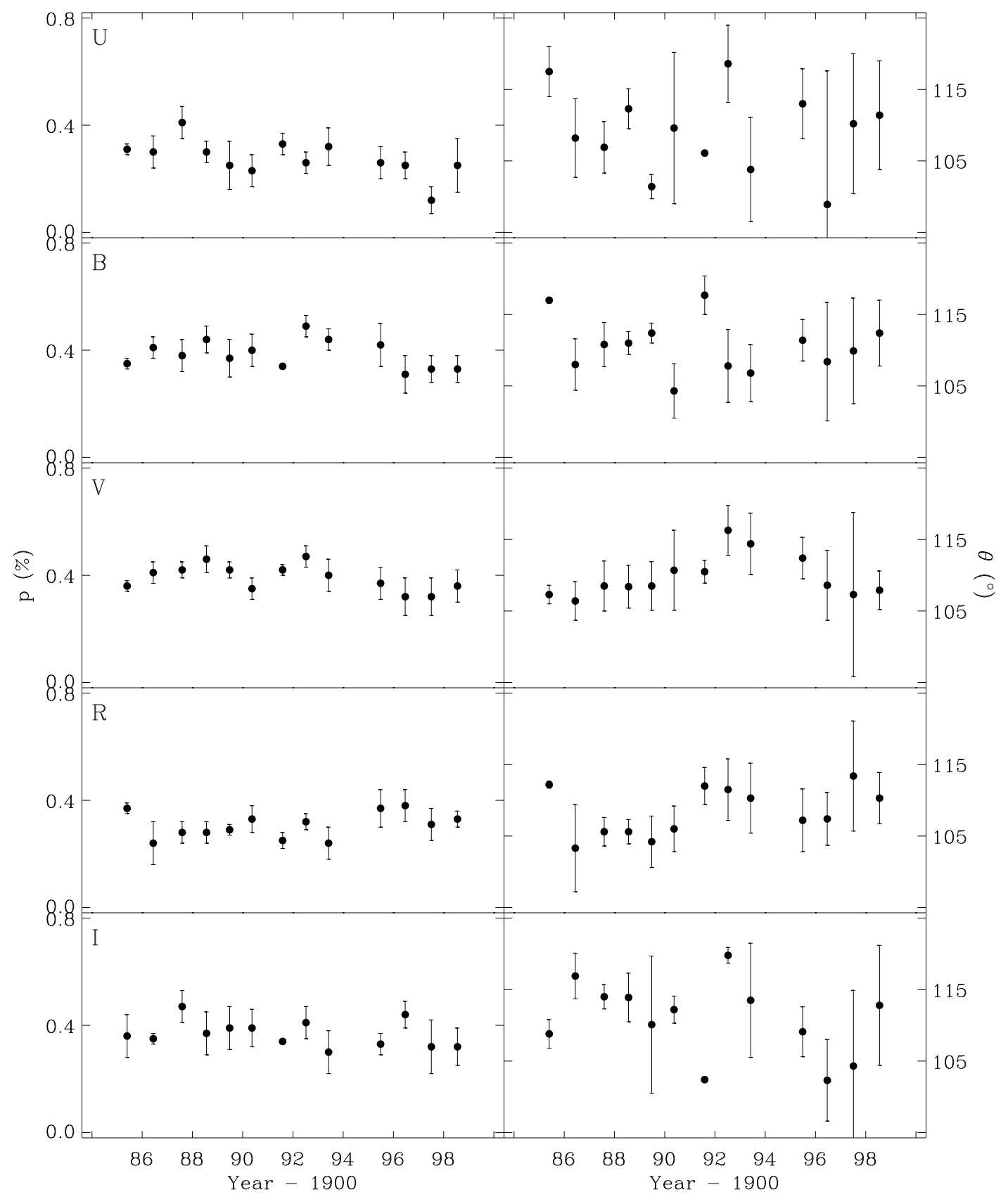



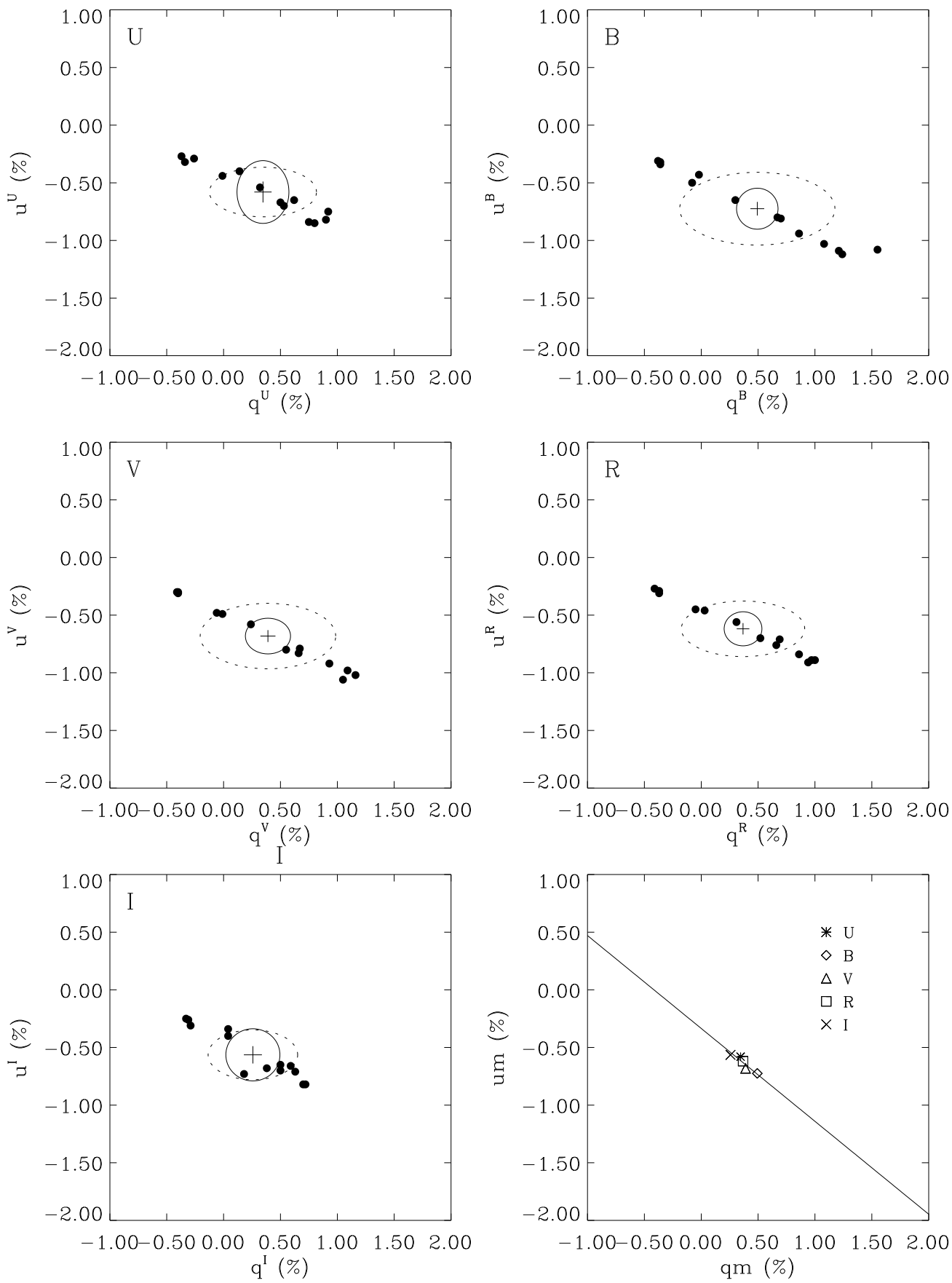


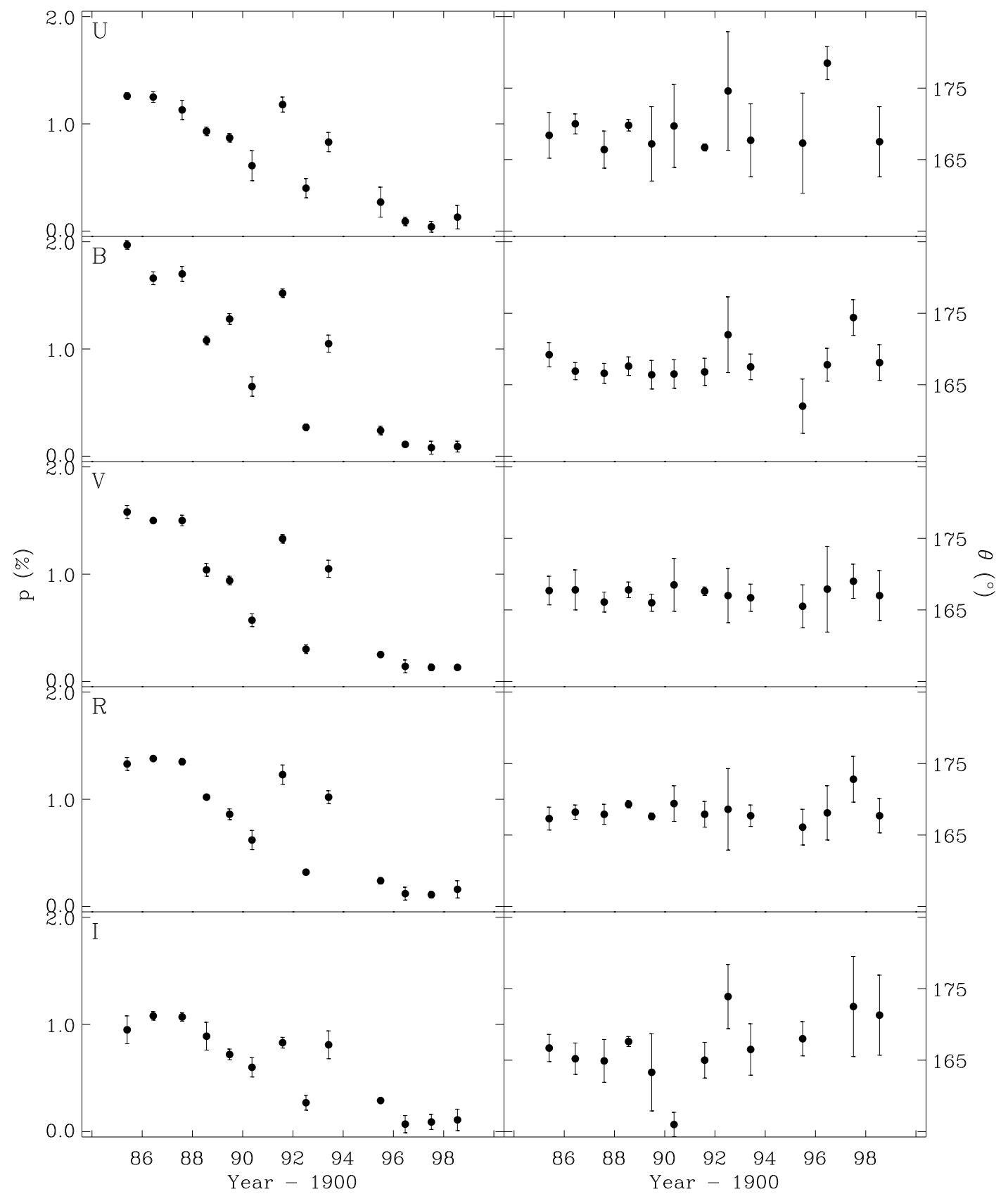



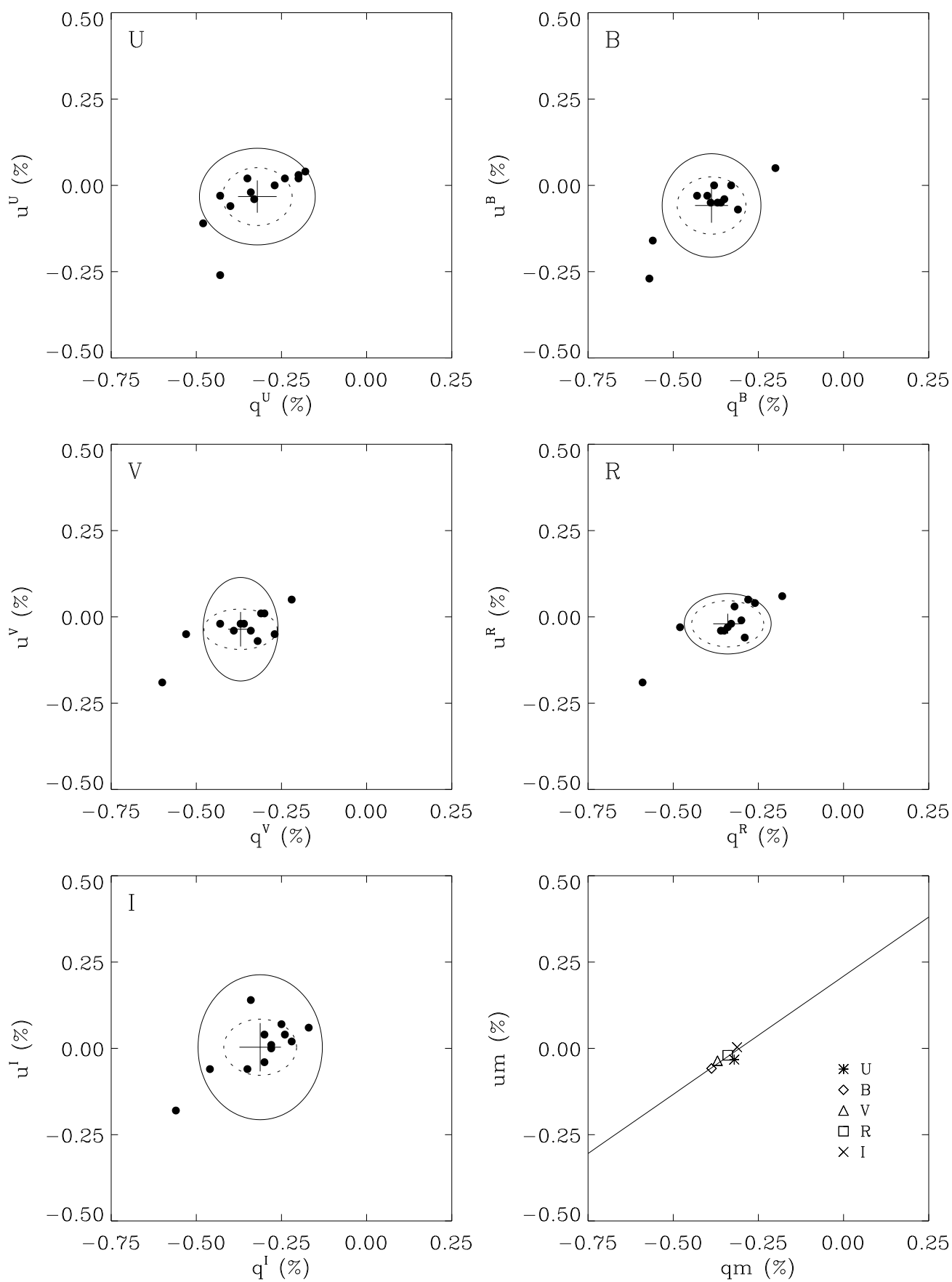


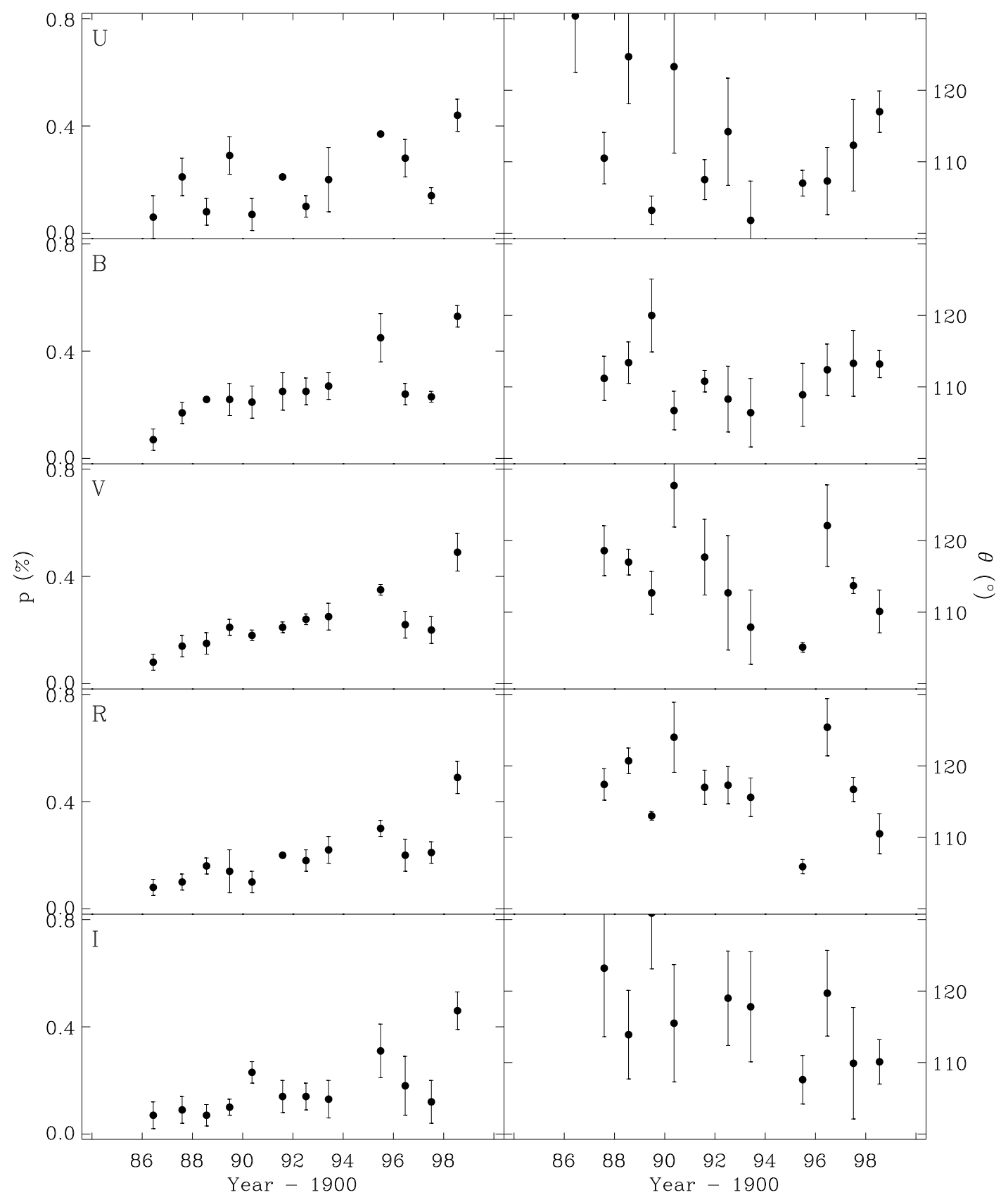

